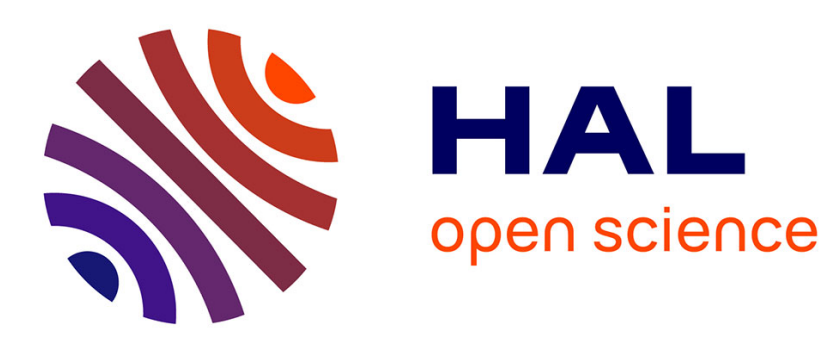

\title{
Power Control and Cell Discontinuous Transmission used as a means of decreasing Small-Cell Networks' Energy Consumption
}

Rémi Bonnefoi, Christophe Moy, Jacques Palicot

\section{To cite this version:}

Rémi Bonnefoi, Christophe Moy, Jacques Palicot. Power Control and Cell Discontinuous Transmission used as a means of decreasing Small-Cell Networks' Energy Consumption. IEEE Transactions on Green Communications and Networking, 2018, 2 (4), pp.899-914. 10.1109/TGCN.2018.2838759 . hal-01798963v4

\section{HAL Id: hal-01798963 \\ https://hal.science/hal-01798963v4}

Submitted on 7 Oct 2018

HAL is a multi-disciplinary open access archive for the deposit and dissemination of scientific research documents, whether they are published or not. The documents may come from teaching and research institutions in France or abroad, or from public or private research centers.
L'archive ouverte pluridisciplinaire HAL, est destinée au dépôt et à la diffusion de documents scientifiques de niveau recherche, publiés ou non, émanant des établissements d'enseignement et de recherche français ou étrangers, des laboratoires publics ou privés. 


\title{
Power Control and Cell Discontinuous Transmission used as a means of decreasing Small-Cell Networks' Energy Consumption
}

\author{
Rémi Bonnefoi, Student Member, IEEE, Christophe Moy, Member, IEEE, and Jacques Palicot, Member, IEEE
}

\begin{abstract}
In this article, we analyze the issues of power control and Cell Discontinuous Transmission (Cell DTx) in a small-cell network. More precisely, we evaluate the performance of a policy, thanks to which each base station minimizes its own energy consumption. We have recently proposed this policy in a monocell scenario and we extend the scope of our previous work in this article, as we propose to use this policy in a multi-cell network. For that purpose, we first consider a network made of two base stations, in which we can compute the minimum network's energy consumption, which enables to provide users with the requested quality of service. If we compare the proposed policy with this minimum, we can demonstrate that the proposed policy has a good performance, which, however, can be improved by reducing the maximum transmit power of the base station. Then, we apply our algorithm in the case of a dense network, in which the proposed policy outperforms other policies and provides a $6 \%$ gain in energy, where compared to a policy featuring no power control. We show that the reduction of the maximum transmit power can increase this gain up to $11 \%$ of the network's energy consumption.
\end{abstract}

Index Terms-Cell Discontinuous Transmission (DTx), Power Control, Green Networks, Small-Cell Networks

\section{INTRODUCTION}

The deployment of dense small-cell networks allows mobile operators to support the ever-increasing demand for mobile data traffic. However, the mobile network densification can increase the circuit power consumed by the network and, consequently, the network's energy consumption. Moreover, nowadays, base stations are responsible for around $50 \%$ of the total carbon emissions of mobile networks [1]. Therefore, improving base stations' energy efficiency has a direct positive effect on the carbon footprint of mobile networks. Moreover, the energy cost has become the predominant operating cost for mobile operators. So decreasing energy consumption is both an economic and ecological issue.

Sleep mode techniques can reduce the energy consumption of the base stations. A first-line solution consists in adapting the set of active base stations to the number of users [2]. In that case, base stations are switched off or put into deep sleep mode for long periods of time, i.e., at least a few minutes [3]. The

R. Bonnefoi and J. Palicot are with CentraleSupélec/IETR, CentraleSupélec campus de Rennes, 35510 Cesson-Sévigné, France email: remi.bonnefoi@centralesupelec.fr, jacques.palicot@centralesupelec.fr. C. Moy is now with Univ Rennes, CNRS, IETR-UMR 6164, F-35000, Rennes, France. e-mail christophe.moy@univ-rennes1.fr.

Part of this work is supported by the project SOGREEN (Smart pOwer GRid for Energy Efficient small-cell Networks), which is funded by the French National Research Agency, under the grant agreement coded: N ANR-14CE28-0025-02 and by Région Bretagne, France. base station selection can be done by means of centralized [4] or decentralized solutions [5]. When some base stations are switched-off, the coverage of the neighboring cells must be extended. This is called cell breathing [6]. The main drawback of this solution is the restart delay.

So, another solution consists in using Cell Discontinuous Transmission (Cell DTx) [7], [8] which means switching a base station to micro-sleep mode for very short periods of time, i.e., a few milliseconds. During these micro-sleeps, some of the elements of the RF chain, such as the power amplifier, are switched-off [9]. In the case of Cell DTx, the power and resource allocations are made in such a manner that some resource elements (subframes) can be freed up, and the base station then switches to micro-sleep mode in order to save energy. This solution is to be used in the LTE standard [10]. Indeed, in this standard, a frame is divided into ten one millisecond-long subframes, and the base station is proposed to be switched to sleep mode during up to six of these subframes.

Furthermore, in cellular networks, the problem pertaining to the power and resource allocation is generally solved in two steps [11], [12]. The issue of power control is first considered. This problem is solved thanks to the computation of the transmit power, the bandwidth and the time during which each user is served. In the LTE standard, this step consists in adjusting the transmit power and finding the number of resource blocks used for each mobile device. Then, the issue of resource allocation is solved and the resource elements, e.g., the resource blocks, are assigned to the users.

In this article, we focus on the problem of power control for the downlink of small-cell networks featuring Cell DTx in Time Division Multiple Access (TDMA). In other words, we focus on the computation of both the transmit power and that of the time during which each user is served. We do not consider the schedule of the users at that stage. On the one hand, poor power control in dense networks greatly increases Inter-cell Interference (ICI) and reduces consequently the users' Signal-to-Interference-plus-Noise Ratio (SINR). On the other hand, where Cell DTx, is used, the power control influences the sleep mode duration. Indeed, for a given quality of service (QoS) (e.g. a capacity constraint), the longer the duration of sleep mode is, the higher the transmit power has to be, where the base station is active.

The problem of power control associated with Cell DTx was first studied in TDMA in a mono-cell scenario in [13]. In [14], the authors use the $\mathcal{W}$-Lambert function [15] to derive a closed-form expression for the optimal number of 
resource blocks that has to be used so as to provide each user with Cell DTx. However, the authors did not consider all the constraints of the problem. In [16], [17], the authors used this closed-form to propose an efficient algorithm, in order to solve the problem of power control associated with Cell DTx. The problem of power control associated with Cell DTx in a mono-cell scenario has also been studied in the Frequency Division Multiple Access (FDMA) case in the literature. A sub-optimal power control was proposed in [18] and the issue of optimal power and resource allocation in FDMA was recently studied in [19], [20]. In all these articles, only one base station has been considered, whereas, in this paper, we study the problem of power control associated with Cell DTx in small-cell networks.

Cell DTx has already been studied in multi-cell scenarios. In [21]-[23], the authors use stochastic geometry tools to analyze the performance of Cell DTx in dense networks. However, in these three papers, the power control is not considered and the transmit power is constant. In [24], [25], the authors proposed efficient resource allocation strategies for Cell DTx in femtocell networks, but the authors did not consider the issue of power control. In [26], [27], the authors studied the issue of the base station's density pertaining to the Cell DTx case. In these two papers, a simple distance-based power control policy is used. Unlike [21]-[27] in which the analysis of Cell DTx is conducted considering a given power control policy, the aim of the present article is to find an efficient power control policy for Cell DTx. In this article, we propose a scheme, in which each base station applies the power control policy that minimizes its own energy consumption. This strategy has been applied in [28]. In this article, the authors propose an algorithm to minimize the energy consumption under delay constraints. Unlike what has been done in [28], in the present article, we consider the issue of energy consumption minimization under capacity constraint. This change in the QoS metric transforms the problem structure and the resolution algorithm. An approach similar to the one proposed herein has been studied in [29]. However, the authors assume that each base station serves only one user, whereas, in the present article, we consider that a base station can serve several users.

In [17], we proposed the first algorithm that uses closedform expressions, in order to solve the issue of power control associated with Cell DTx in TDMA. This solution was proposed in a mono-cell scenario, but can be used in a multi-cell network. In that case, each base station optimizes its own energy consumption. The aim of this article is to extend the use of this power control policy in a multi-cell network and to evaluate its performance in such a network. To do so, we first consider a network composed of two base stations. In this network, we can compute the minimal base station's power consumption, which enables to serve the users. This minimum provides a lower-bound which is used as a baseline in order to evaluate the proposed policy. We show that the proposed solution has a good performance, which, however, can be improved by means of reducing the maximum transmit power of the base stations. Our results show that this reduction of the maximum transmit power does indeed improve the performance of the proposed solution. Besides, we

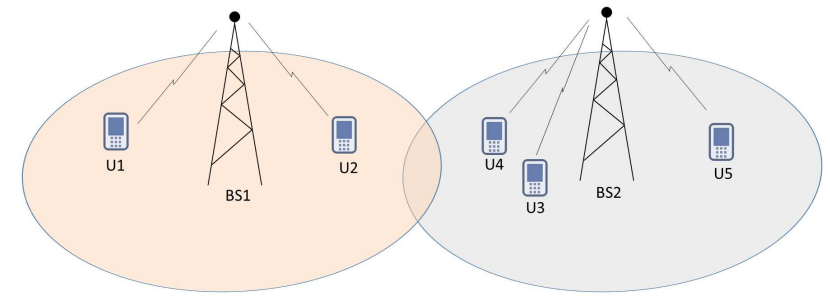

Fig. 1. System model with two base stations which serve the users in their cell coverage. In this scheme, $N_{B S}=2, N_{u}=5, S_{1}=\{1,2\}$ and $S_{2}=\{3,4,5\}$. The base stations adapt the transmit power and the proportion of time used to serve the users.

investigate the value of the optimal transmit power. Then, we use numerical simulations and analytical derivations in order to show that all the results, obtained in a network composed of two base stations, remain valid when the number of base station increases.

The rest of this article is organized as follows. The system model is introduced in Section II. We present the proposed policy in Section III. In Section IV, we study the minimum base station's power consumption in a network made of two base stations. We assess the performance of the proposed policy in such a network in Section V. In Section VI, we show that the performance of the proposed policy can be improved by reducing the maximum RF transmit power of the base station. In Section VII, we extend the use of the proposed solution to dense networks. Then, in Section VIII, we investigate the optimal value for the maximum transmit power in a dense network and Section IX concludes this article.

\section{SySTEM MODEL}

In this article, as illustrated in Figure 1, we consider the downlink of $N_{B S}$ small-cells, which serve $N_{u}$ users. Orthogonal Frequency Division Multiplexing (OFDM) is used by the base stations. Small-cells are perfectly synchronized, and we do not consider any coordination between them. We denote $k \in \llbracket 1 ; N_{u} \rrbracket$ the users' index and $n \in \llbracket 1 ; N_{B S} \rrbracket$ denotes the index of base stations. In this article, $\llbracket 1 ; N \rrbracket$ denotes $\{1, \cdots, N\}$, the set of integers between 1 and $N$. Besides, $S_{n}$ is the set of users served by base station $n$ and $N_{n}=\left|S_{n}\right|$ denotes its cardinality.

Moreover, we suppose a linear model for the base station's power consumption [30]:

$$
\left\{\begin{array}{cccc}
P_{\text {supply }} & = & P_{0}+m_{p} P_{T_{X}} & \text { when active, } \\
P_{\text {supply }} & = & P_{S} & \text { during sleep mode. }
\end{array}\right.
$$

Where $P_{0}$ is the static power consumption where the base station is active (processing, cooling, etc). $P_{s}$ is the base station's power consumption during sleep mode, $m_{p}$ is the coefficient of the load dependence. $P_{T_{X}} \in\left(0 ; P_{\max }\right]$ is the instantaneous RF transmit power. $P_{\max }$ denotes the maximum RF transmit power of the base station. According to [30], for a small-cell, $P_{0}=56 \mathrm{~W}, P_{s}=39 \mathrm{~W}, P_{\max }=6.3 \mathrm{~W}$, and $m_{p}=2.6$.

Moreover, we consider flat fading channels, and the pathloss between the base station $n$ and the user $k$ is denoted $G_{n, k}$. Besides, $N=k_{B} T_{0} B$ denotes the thermal noise which is the product of the bandwidth $B$, the Boltzmann constant $k_{B}$ and the temperature $T_{0} . I_{k}$ is the interference generated by the 


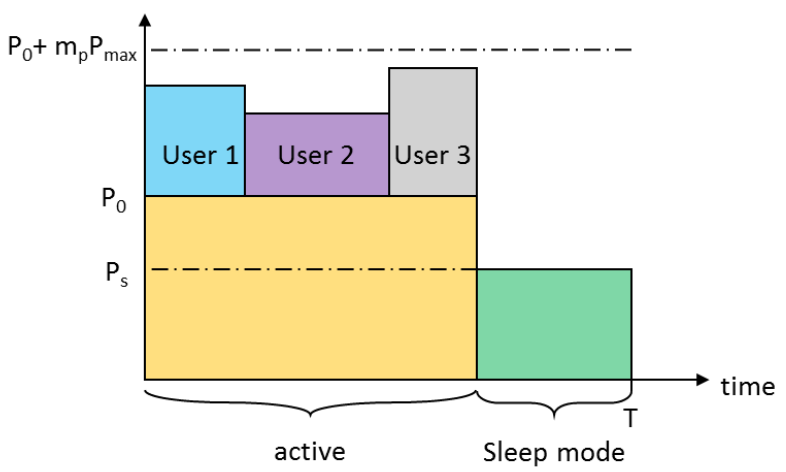

Fig. 2. Variation of the base station power consumption during a frame. The base station serves $N_{n}=3$ users and then switches to sleep mode until the end of the frame.

neighboring cells of the user $k$. We suppose that each user is served by the base station that features the lowest path-loss.

Furthermore, each base station provides each user with a given capacity $C_{k}$. As illustrated in Figure 2, we suppose that, in each frame of length $T$, the base station $n$ serves $N_{n}$ users and then switches to sleep mode in order to save energy. As in [13], [17], we suppose that TDMA is used by the base station to serve the users.

In each frame of duration $T$, we denote $t_{n k}$ the time during which the base station $n$ serves the user $k \cdot \mu_{n k}=\frac{t_{n k}}{T}$ is the proportion of the frame used to serve this user. In addition, the transmit power used for the user $k$ is denoted $P_{T_{X}}^{n k}$ in this article.

All the base stations in the network use the same channel for downlink communications. As a consequence, the users experience the interference generated by the neighboring base stations. We denote $I_{k}$ the interference generated by the neighboring cells on the user $k$ :

$$
I_{k}=\sum_{m=1, m \neq n}^{N_{B S}} I_{m \rightarrow k}
$$

Where $I_{m \rightarrow k}$ is the interference generated by the base station $m$ on the user $k$. In this article, we do not consider the issue of resource allocation and focus on the issue of power control. In order to make the power control policy independent from the resource allocation strategy, as in [26], [27], we suppose that each user experiences the interference generated by the time average transmit power of the neighboring base stations:

$$
I_{m \rightarrow k}=G_{m k} \sum_{l \in S_{m}} \mu_{m l} P_{T_{X}}^{m l}
$$

Moreover, we suppose that, the rate of a user is equal to the Shannon's capacity when the user is served and equal to zero during the rest of the frame. With this assumption, the average rate of the user $k$ during a frame is equal to [13]:

$$
\begin{aligned}
C_{k} & =B \mu_{n k} \log _{2}\left(1+\frac{P_{T_{X}}^{n k} G_{n k}}{N+I_{k}}\right) \\
& =B \mu_{n k} \log _{2}\left(1+\frac{P_{T_{X}}^{n k} G_{n k}}{N+\sum_{m=1, m \neq n}^{N_{B S}} \sum_{l \in S_{m}} G_{m k} \mu_{m l} P_{T_{X}}^{m l}}\right) .
\end{aligned}
$$

\section{PROPOSED POLICY: THE OPTIMAL MONO-CELL POLICY}

\section{A. Problem formulation}

In this section, we introduce an algorithm that solves the problem of power control associated with Cell DTx. According to this policy, each base station adjusts its transmit power and sleep mode duration, in order to minimize its energy consumption while satisfying, for each user, a capacity constraint. This optimization is carried out without any cooperation with the neighboring base stations. This policy has been introduced in [17], but the intercell interference was not considered then. The policy proposed here, only requires the base station $n$ to know the value of $\frac{G_{n k}}{N+I_{k}}$ so as to serve the user $k$. This value can be transmitted to the base station by the users. Indeed, in each frame, each user can use the pilot symbols in order to evaluate the average noise-plus-interference level and path-loss between the base station and them. Then, the user sends the ratio between these two averages to the base station via the uplink channels. The value of $\frac{G_{n k}}{N+I_{k}}$ is then used by the base station to compute the transmit power and service time for each user. Please, note that, in this article, we focus on the energy consumption of downlink communications, and we do not consider the energy consumed by this uplink communications.

In order to minimize the base station's energy consumption, we minimize the average base station's power consumption during a frame. We obtain the following optimization problem:

$$
\begin{aligned}
\min _{\mu_{n k}, P_{T_{X}}^{n k}} & P_{s}+\left(P_{0}-P_{S}\right) \sum_{k \in S_{n}} \mu_{n k}+m_{p} \sum_{k \in S_{n}} \mu_{n k} P_{T_{X}}^{n k}, \\
\text { s.t. } & P_{T_{X}}^{n k} \leq P_{\max }, \quad \forall k \in S_{n}, \\
& \mu_{n k} \geq 0, \quad \forall k \in S_{n}, \\
& \sum_{k \in S_{n}} \mu_{n k} \leq 1, \\
& C_{k}=B \mu_{n k} \log _{2}\left(1+\frac{P_{T_{X}}^{n k} G_{n k}}{N+I_{k}}\right), \quad \forall k \in S_{n} .
\end{aligned}
$$

In equation (5), equation (5a) is the average base station's power consumption during a frame. The minimization of this function is done while considering the constraints of the system: the user's capacity constraint must be satisfied (equation (5e)), the user's service time must be positive (equation $(5 \mathrm{c})$ ), the total service time must be lower than the frame duration (equation (5d)), and the instantaneous transmit power must be lower than the maximum transmit power of the base station denoted $P_{\max }$ (equation (5b)).

\section{B. Problem reformulation}

The problem of equation (5) depends on two types of variables: the service times $\mu_{n k}$ and the transmit powers $P_{T_{X}}^{n k}$. In order to ease the resolution, we rewrite this problem as a function of the service times $\mu_{n k}$ only. The expression of the transmit power, which provides the user $k$ with the required capacity, is:

$$
P_{T_{X}}^{n k}=\frac{N+I_{k}}{G_{n k}}\left(2^{\frac{C_{k}}{B \mu_{n k}}}-1\right) .
$$


Equation (6) allows to rewrite equation (5a) as a function of the service times only. Moreover, the constraint on the maximum transmit power of equation (5b) can also be rewritten as a constraint on the service time. Indeed, providing the user $k$ with an RF transmit power that is lower than $P_{\max }$, is equivalent to serve the said user during a time that is longer than a minimum service time, denoted $\mu_{n k}^{\min } \cdot \mu_{n k}^{\min }$ is the time that would be used by the base station to serve the user $k$ with the maximum transmit power:

$$
\mu_{n k}^{\min }=\frac{C_{k}}{B \log _{2}\left(1+\frac{P_{\max } G_{n k}}{N+I_{k}}\right)},
$$

If $\mu_{n k}<\mu_{n k}^{\min }$, then $P_{T_{X}}^{n k}>P_{\max }$ and the value of $\mu_{n k}$ will be inoperable in practice. We can finally rewrite this optimization problem as a function of the service times $\mu_{n k}$ only:

$$
\begin{gathered}
\min _{\mu_{n k}} P_{s}+\sum_{k \in S_{n}} \mu_{n k}\left[\left(P_{0}-P_{s}\right)+m_{p} \frac{N+I_{k}}{G_{n k}}\left(2^{\frac{C_{k}}{B \mu_{n k}}}-1\right)\right], \\
\text { s.t. } \mu_{n k} \geq \mu_{n k}^{\min }=\frac{C_{k}}{B \log _{2}\left(1+\frac{G_{n k} P_{\max }}{N+I_{k}}\right)}, \quad \forall k \in S_{n} \\
\quad \sum_{k \in S_{n}} \mu_{n k} \leq 1 .
\end{gathered}
$$

Furthermore, we can see in equation (8a) that the average base station's power consumption is the sum of two terms: the static and dynamic power consumptions. The expression of the average static power consumption of the base station is:

$$
P_{\text {stat }}=P_{S}+\left(P_{0}-P_{S}\right) \sum_{k \in S_{n}} \mu_{n k},
$$

and the expression of the dynamic power consumption is:

$$
P_{\mathrm{dyn}}=m_{p} \sum_{k \in S_{n}} \mu_{n k} \frac{N+I_{k}}{G_{n k}}\left(2^{\frac{C_{k}}{B \mu_{n k}}}-1\right) .
$$

Finding a trade-off between the static and dynamic power consumptions is a requirement so as to mitigate the network's energy consumption.

\section{Proposed Policy}

We have shown in [16] that the problem of equation (8a) is convex. We can consequently use the Karush-Kuhn-Tucker conditions to solve it. With these conditions, each constraint can either be binding or non-binding, i.e., can be considered as an equality or as a strict inequality. We consequently have different cases, depending on the binding constraints. We first derive the value of $\mu_{n k}$, for which the gradient of equation (8a) is equal to zero, and we then obtain [17]:

$$
\mu_{n k}^{o p t}=\frac{C_{k} \log (2)}{B} \frac{1}{W\left(e^{-1}\left(\frac{G_{n k}}{N+I_{k}} \frac{P_{0}-P_{s}}{m_{p}}-1\right)\right)+1},
$$

where $W$ denotes the $W$-Lambert function which is the reciprocal bijection of $f(x)=x e^{x}$ over $\left[-e^{-1} ;+\infty\right)$. This function verifies $x=f^{-1}\left(x e^{x}\right)=\mathcal{W}\left(x e^{x}\right)$ [15]. Once this expression has been established, we have to verify if the constraints are binding or not. Indeed, the value of $\mu_{n k}^{o p t}$ given

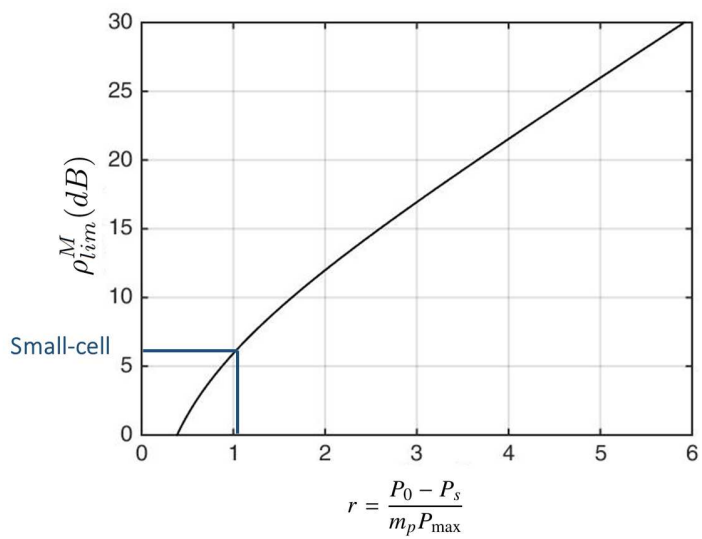

Fig. 3. In this figure, we display $\rho_{\lim }^{M}(r)$ that determines the value of $\rho_{\mathrm{lim}}^{\boldsymbol{M}}$ for any base station type. For a base station type, i.e., a set of parameters $P_{0}$, $P_{s}, m_{p}$ and $P_{\max }$ we can calculate the value of $r$. Thanks to this figure we can find the corresponding value of $\rho_{\lim }^{\boldsymbol{M}}$ as we did for a small-cell. Then, for any user served by this base station, if $\rho_{n k}^{M} \geq \rho_{\text {lim }}^{M}$ its service time has to be computed with equation (11), else with equation (7)

in equation (11) is optimum where none of the constraints are binding. The first set of constraints to be considered are the ones given on the minimum service time of equation ( $8 b$ ). In order to consider these constraints, we introduce two variables. We denote $\rho_{n k}^{M}$, the SINR of the user $k$ if he/she was provided with the maximum RF transmit power $P_{\max }$ :

$$
\rho_{n k}^{M}=\frac{P_{\max } G_{n k}}{N+I_{k}} .
$$

The second variable introduced here is the ratio $r$, which is the ratio between the variation range of respectively the static and the dynamic power consumptions:

$$
r=\frac{P_{0}-P_{s}}{m_{p} P_{\max }} .
$$

The numerator of this quantity is the difference between the maximum and the minimum static power consumptions of the base station, i.e., the difference between $P_{0}$ and $P_{s}$. Similarly, the denominator of this quantity is the difference between the maximum and minimum dynamic power consumption, which lies in $\left(0 ; m_{p} P_{\max }\right]$. It has been proved in [17] that the choice between $\mu_{n k}^{\min }$ and $\mu_{n k}^{o p t}$, respectively defined by (7) and (11), can be done by means of the comparison of $\rho_{n k}^{M}$ with a threshold $\rho_{\lim }^{M}(r)$, which depends only on the ratio $r$. In other words, we know whether a user has to be provided with a transmit power equal to $P_{\max }$ during $\mu_{n k}^{\min }$ or with a lower transmit power during $\mu_{n k}^{o p t}$, only thanks to the comparison of between $\rho_{n k}^{M}$ and $\rho_{\mathrm{lim}}^{M}(r)$.

The function $\rho_{\lim }^{M}(r)$ is displayed in Figure 3. For a given base station type, i.e., a set of parameters $P_{0}, P_{s}, P_{\max }$ and $m_{p}$, there is a specific SINR value denoted $\rho_{\mathrm{lim}}^{M}$, under which a user has to be served with the maximum transmit power $P_{\max }$ during the minimum time $\mu_{n k}^{\min }$. The limit $\rho_{\text {lim }}^{M}$ depends only on the ratio $r$ of the base station. In other words, if a user has a high SINR $\rho_{n k}^{M}$, he is served with a low transmit power and the service time is computed with (11). The transmit power used for a user increases as $\rho_{n k}^{M}$ decreases. When $\rho_{n k}^{M}$ becomes lower than $\rho_{\text {lim }}^{M}$, the transmit power must be set to $P_{\max }$, and the user's service time is computed thanks to equation (7). We 
can see in Figure 3 the value of $\rho_{\lim }^{M}$ for a small-cell. For this base station type, only users with a low value of $\rho_{n k}^{M}$, below $\rho_{\lim }^{M} \leq 6.2 \mathrm{~dB}$, are served with the maximum transmit power during $\mu_{n k}^{\min }$. It is worth noting that, for a given value of the parameters $P_{0}, P_{s}$ and $m_{p}$, the value of $\rho_{\text {lim }}^{M}$ increases as $P_{\max }$ decreases.

Once the constraints of equation (8b) have been considered, we have to verify if the total service time is not longer than the frame duration (constraint $(8 \mathrm{c})$ ). If this constraint is not met, the problem has to be solved by considering it binding, i.e., as an equality. In this case, the base station does not have to be switched to sleep mode so as to minimize its energy consumption. It has been shown in [17] that the value of $\mu_{n k}^{o p t}$ can either be equal to:

$$
\mu_{n k}^{o p t}(K)=\frac{C_{k} \log (2)}{B} \frac{1}{W\left(e^{-1}\left(\frac{G_{n k}}{N+I_{k}} \frac{K_{o p t}}{m_{p}}-1\right)\right)+1},
$$

or equal to $\mu_{n k}^{\min }$. In this equation $K_{o p t}$ has to be computed numerically. For that purpose, we first have to identify the set of users served during $\mu_{n k}^{\mathrm{min}}$. Where equations (14) and (11) are compared, $K_{o p t}$ can then be viewed as the theoretical value of $P_{0}-P_{s}$, for which the constraint of equation (8c) is an equality. As a consequence, for any value of $K$ that is lower than $K_{o p t}, \sum_{k \in S_{n}} \mu_{n k}^{o p t}(K)>1$, and for any value of $K$ that is higher than $K_{o p t}, \sum_{k \in S_{n}} \mu_{n k}^{o p t}(K)<1$. Moreover, for each user, there is a value denoted $K_{k}$, from which the user has to be served during $\mu_{n k}^{\min }$. This value verifies $\mu_{n k}^{\text {opt }}\left(K_{k}\right)=\mu_{n k}^{\min }$ and is equal to:

$$
K_{k}=m_{p} P_{\max }\left[\left(\frac{N+I_{k}}{G_{n k} P_{\max }}+1\right) \ln \left(1+\frac{P_{\max } G_{n k}}{N+I_{k}}\right)-1\right] .
$$

An analysis of the derivative shows that $K_{k}$ increases as $\frac{G_{n k}}{\left(N+I_{k}\right)}$ increases. As a consequence, if a user has to be served during $\mu_{n k}^{\min }$, then all users featuring a lower SINR have to be served during $\mu_{n k}^{\mathrm{min}}$. Finally, we have to find the user featuring the highest value of $\frac{G_{n k}}{\left(N+I_{k}\right)}$, which is served during $\mu_{n k}^{\min }$. If we denote the user $k$ as being this user, we have, $\sum_{l \in S_{n}} \mu_{n l}^{o p t}\left(K_{k}\right)>1$, and for the user whose SINR is directly higher (namely user $k+1), \sum_{l \in S_{n}} \mu_{n l}^{o p t}\left(K_{k+1}\right)<1$ ). As a consequence, the set of users served during the minimum service time can be computed by evaluating the sums $\sum_{l \in S_{n}} \mu_{n l}^{o p t}\left(K_{k}\right)$ for all users. If this sum is greater than 1 , the user $k$ has to be served during $\mu_{n k}^{\min }$, otherwise its service time can be computed with equation (14). Once the set of users served during $\mu_{n k}^{\min }$ has been identified, we can compute a numerical approximation of $K_{o p t}$, which is the solution of:

$$
\sum_{k=1}^{N_{n}^{\min }} \mu_{n k}^{\min }+\sum_{k=N_{n}^{\min }+1}^{N_{n}} \mu_{n k}^{o p t}(K)-1=0
$$

where $N_{n}^{\min }$ denotes the set of users which have to be served during $\mu_{n k}^{\min }$. Equation (16) can be solved using a single variable root finding algorithm such as the Newton method [31].

\section{OPTIMAL POLICY WITH A PERFECT CSI: A LOWER-BOUND FOR THE POWER CONSUMPTION}

In Section V, we assess the performance of the policy introduced in Section III in a network made of two base stations. Before making such an assessment, we need some reference policies. In particular, in a network made of two base stations, we can numerically compute the minimum average power consumption, which enables to satisfy the users' capacity constraints. This minimum provides a lowerbound for the performance of the studied policy in a network composed of two cells.

In a network composed of $N_{B S}$ base stations, minimizing the network's energy consumption is equivalent to solving the following problem:

$$
\begin{array}{cl}
\min _{\mu_{n k}, P_{T_{X}}^{n k}} & N_{B S} P_{S}+\sum_{n=1}^{N_{B S}} \sum_{k \in S_{n}} \mu_{n k}\left[\left(P_{0}-P_{S}\right)+m_{p} P_{T_{X}}^{n k}\right], \\
\text { s.t. } \quad & P_{T_{X}}^{n k} \in\left(0 ; P_{\max }\right], \quad \forall k \in \llbracket 1 ; N_{u} \rrbracket, \\
C_{k}= & B \mu_{n k} \log _{2}\left(1+\frac{P_{T_{X}}^{n k} G_{n k}}{N+I_{k}}\right), \quad \forall k \in \llbracket 1 ; N_{u} \rrbracket, \\
& \mu_{n k}>0, \quad \forall k \in \llbracket 1 ; N_{u} \rrbracket, \\
& \sum_{k \in S_{n}} \mu_{n k} \leq 1, \quad \forall n \in \llbracket 1 ; N_{B S} \rrbracket .
\end{array}
$$

Solving this problem requires the knowledge of all the path-losses that exist between the users and the base stations. Furthermore, it is essential to find the value of the transmit powers $P_{T_{X}}^{n k}$ and service times $\mu_{n k}$, which provide each user with their capacity constraint (equation (17c)). This problem must be solved while considering the constraints of the system. The transmit power has to be lower than the maximum RF transmit power of the base station (constraints of equation (17b)) and the total service time of a base station must be lower than the frame duration (equation (17e)).

In that form, the problem set in equations (17) is not convex [32]. However, in the case where $N_{B S}=2$, this problem can be rewritten in a convex form. For that purpose, we introduce the following variables:

$$
x_{k}=\mu_{n k} P_{T_{X}}^{n k}, \quad \forall k \in \llbracket 1 ; N_{u} \rrbracket,
$$

where $x_{k}$ is the average transmit power used by the base station $n$ to serve the user $k$ in a frame. This value is computed thanks to the division of the RF energy used by the base station to serve the user $k$, by the frame duration $T$. Furthermore, in the case of two base stations, the expression of the users' capacity constraints can be rewritten:

$$
C_{k}=B \mu_{n k} \log _{2}\left(1+\frac{P_{T_{X}}^{n k} G_{n k}}{N+G_{m k} \sum_{l \in S_{m}} x_{l}}\right), \quad \forall k \in \llbracket 1 ; N_{u} \rrbracket .
$$

For $n \in\{1 ; 2\}$ and, $m=1+(n \bmod 2)$, for $k \in \llbracket 1 ; N_{u} \rrbracket$, we can deduce from equation (19):

$$
P_{T_{X}}^{n k}=\left(2^{\frac{C_{k}}{B \mu_{n k}}}-1\right)\left(\frac{N}{G_{n k}}+\frac{G_{m k}}{G_{n k}} \sum_{l \in S_{m}} x_{l}\right), \quad \forall k \in \llbracket 1 ; N_{u} \rrbracket .
$$


If we multiply each equation of (20) by $\mu_{n k}$, we obtain the following system of linear equations in $x_{k}$ :

$$
x_{k}-\alpha_{k} \sum_{l \in S_{m}} x_{l}=\beta_{k}, \quad \forall k \in \llbracket 1 ; N_{u} \rrbracket,
$$

where,

$$
\alpha_{k}=\frac{G_{m k}}{G_{n k}} \mu_{n k}\left(2^{\frac{C_{k}}{B \mu_{n k}}}-1\right),
$$

and,

$$
\beta_{k}=\frac{N \mu_{n k}\left(2^{\frac{C_{k}}{B \mu_{n k}}}-1\right)}{G_{n k}} .
$$

Solving this linear system yields the expression of $x_{k}$ as being a function of the variables $\mu_{n l}, \forall l \in \llbracket 1 ; N_{u} \rrbracket$. After some mathematical derivations detailed in Appendix A, we obtain the expression of $x_{k}$ as a function of the service times $\mu_{n l}$.

$$
\begin{aligned}
& x_{k}=\quad \underbrace{\beta_{k}} \\
& \text { Average transmit power } \\
& \text { used for user } k \text { without ICI } \\
& +\underbrace{}_{k\left[\frac{\sum_{i \in S_{n}} \sum_{j \in S_{m}} \alpha_{j} \beta_{i}+\sum_{j \in S_{m}} \beta_{j}}{1-\sum_{i \in S_{n}} \sum_{j \in S_{m}} \alpha_{i} \alpha_{j}}\right]}, \\
& \text { Extra power consumed because of ICI }
\end{aligned}
$$

where $m=1+(n \bmod 2)$. We can see in equation (22) that $x_{k}$ is the sum of two terms. The first one is the average transmit power, which is necessary, where there is no ICI. The second term is the RF power spent to tackle the inter-cell interference.

Moreover, if we introduce the value of $x_{k}$ of equation (22) in the optimization problem of (17), we then obtain a minimization problem that only depends on a single type of variable: the service times $\mu_{n l}$. When rewriting the problem, the constraint reformulation has to be done carefully, especially with regard to the constraints on the transmit power (i.e., equation (17b)). According to this constraint, a solution can be found if and only if $P_{T_{X}}^{n k}>0, \forall k \in \llbracket 1 ; N_{u} \rrbracket$. In other words, as $\mu_{n k}>0, \forall k \in \llbracket 1 ; N_{u} \rrbracket$, a solution can be found if and only if $x_{k}>0$ for all $k$, and consequently only if $\sum_{k=1}^{N_{u}} x_{k}>0$. The expression of $\sum_{k=1}^{N_{u}} x_{k}>0$ is:

$$
\begin{aligned}
\sum_{k=1}^{N_{u}} x_{k}=\frac{\sum_{i \in S_{1}} \beta_{i}+\sum_{i \in S_{1}} \sum_{j \in S_{2}} \alpha_{i} \beta_{j}}{1-} & \sum_{i \in S_{1}} \sum_{j \in S_{2}} \alpha_{i} \alpha_{j} \\
+ & \frac{\sum_{i \in S_{1}} \sum_{j \in S_{2}} \alpha_{j} \beta_{i}+\sum_{j \in S_{2}} \beta_{j}}{1-\sum_{i \in S_{1}} \sum_{j \in S_{2}} \alpha_{i} \alpha_{j}}>0 .
\end{aligned}
$$

Equation (23) shows that a solution can be reached only if:

$$
\sum_{i \in S_{1}} \sum_{j \in S_{2}} \alpha_{i} \alpha_{j}<1
$$

Conversely, according to equation (22), if $\sum_{i \in S_{1}} \sum_{j \in S_{2}} \alpha_{i} \alpha_{j}<1$ and $\mu_{n k}>0, \forall k \in \llbracket 1 ; N_{u} \rrbracket$, then $x_{k}>0, \forall k \in \llbracket 1 ; N_{u} \rrbracket$. As a consequence, in the new formulation of the problem, the inequality $P_{T_{X}}^{n k}>0$ can be replaced by equation (24). With this new constraint, in a network made of two base stations, we can rewrite the problem of equation (17) as:

$$
\begin{array}{ll}
\min _{\mu_{n k}} & 2 P_{s}+\left(P_{0}-P_{S}\right) \sum_{n=1}^{2} \sum_{k \in S_{n}} \mu_{n k}+m_{p} \sum_{n=1}^{2} \sum_{k \in S_{n}} x_{k}, \\
\text { s.t. } & x_{k} \leq \mu_{n k} P_{\max }, \quad \forall k \in \llbracket 1 ; N_{u} \rrbracket, \\
& \sum_{i \in S_{1}} \sum_{j \in S_{2}} \alpha_{i} \alpha_{j}<1, \\
& \mu_{n k}>0, \quad \forall k \in \llbracket 1 ; N_{u} \rrbracket, \\
& \sum_{k \in S_{n}} \mu_{n k} \leq 1, \quad \forall n \in \llbracket 1 ; N_{B S} \rrbracket .
\end{array}
$$

Proposition IV.1. In a network made of two base stations, the problem introduced in equation (25) is convex.

The proof is provided in Appendix B. As the studied problem is convex, numerical methods such as the gradient descent or interior point methods [32] can be used to solve it efficiently.

Solving this problem provides an optimum which cannot be reached in practice as it is impossible to have knowledge of all the information. However, this optimum provides a lower-bound for the average network's power consumption. In Section V, we compute this lower-bound and use it for evaluating the performance of the policy described in Section III.

\section{EVALUATION IN A TWO CELLS NETWORK}

In this section, we assess the performance of the policy described in Section III in a network made of two cells. For that purpose, we consider a network composed of two small cells featuring a $500 \mathrm{~m}$ intersite distance. This network serves several users uniformly distributed in a $1000 \times 500 \mathrm{~m}^{2}$ area. The users are served in a band featuring a $10 \mathrm{MHz}$ bandwidth and a $2 \mathrm{GHz}$ central frequency. The path-losses are computed using the Winner II "Urban micro-cell" (B1) channel model [33].

\section{A. Behavior of the policy in a two-cell scenario}

In the case of the power control policy described in Section III, the power control mechanism is a dynamic process. Indeed, when one of the base station changes its transmit power, it changes the interference in the network and the neighboring base stations adapt their transmit powers and the users' service times. Then, all the base stations (including the first one which changed its transmit power) will increase their transmit power and the users' service times to cope with the additional interference, and so on. Thus, during some frames, all the base stations are adjusting the transmission parameters, and the capacity constraints of the users are not satisfied. As an example, if we suppose that a new user connects to a base station in a two-cell network, the first base station (to which the new user connects) increases its average transmit power and consequently the generated interference. The second base station will increase its transmit power and the users' service times to compensate this increase. In turn, the first base station will increase its transmit power and the users' service time, etc. 
So, there is a transition phase during which the base stations adapt the transmit powers and the users' service times.

In this section, we analyze the transition phase, when a new user connects to the network. A priori, we can be in two different situations:

- If the transition phase, during which base stations adapt their transmit powers, is long, then the proposed solutions cannot be used in a multi-cell network.

- On the contrary, if the transition phase is short, then the proposed solution can be used in a network composed of several cells.

In the following, we use numerical simulations in the network composed of two base stations in order to show that the transition phase during which the base stations adapt their transmit power is short. In this network, we suppose that ten users appear one after the other in the coverage area of the cells and that each of them has a capacity constraint equal to $5 \mathrm{Mbits} / \mathrm{s}$. Moreover, before each frame, the base station recomputes the value of the transmit power and service time of each user. We suppose that a new user connects to the base station, whose associated path-loss is the lowest one, every ten frames. When all the users are connected to the base station, they disconnect one after the other in the reverse order of appearance. Users are indexed by order of appearance, and we display in Figures $4 \mathrm{a}$ and $4 \mathrm{~b}$ the evolution of the transmit power and the service time of users 1, 3 and 5 .

As expected, we can see in Figure 4a that the instantaneous transmit power used to serve each connected user increases when a new user appears. Moreover, once a new user has connected, the transmit powers used converge in less than five frames to a constant value. The same result can be observed regarding the proportions of time used to serve users in Figure 4b. As the transition times are very short, they can be neglected and the performance of this power control policy can be evaluated when all the users are connected.

\section{B. Performances evaluation}

We can now use the minimum previously studied as a lowerbound for the analysis of the policy described in Section III. We consider the network composed of two base stations introduced earlier. The two base stations serve on average 20 users distributed following a Poisson Point Process (PPP). In this network, we compare four different policies:

- The proposed policy presented in Section III.

- The lower-bound introduced in Section IV.

- A policy without any power control. In this policy, each base station serves the users by means of a transmit power equal to $P_{\max }$ during $\mu_{n k}^{\min }$. In this policy, the time during which the base station is switched to sleep mode is maximized.

- A policy without any Cell DTx. In this policy, the base station does not switch to sleep mode during the frame and the power control is done in order to minimize the dynamic power consumption of the base station.

The results are displayed in Figure 5a. We can see that, in our scenario, the proposed policy has always a better performance than the policy without any power control, and than the policy without any Cell DTx. When the users' capacity constraint is low, i.e., below $1 \mathrm{Mbit} / \mathrm{s}$, the proposed policy allows to save $30 \%$ of the average base station's power consumption, in comparison to a strategy without any Cell DTx. Where compared to the policy without any power control, the proposed policy allows to save up to $10 \%$ of the base station's power consumption. In other words, the proposed policy saves up to $40 \%$ of the proportion of power consumption, on which the power control has an effect (total power consumption minus the power consumption during sleep mode $P_{S}$ ).

Moreover, if we compare the proposed policy to the lowerbound, we can see that the gap existing between these two curves increases as the users' capacity constraint rises. If we only consider the proportion of power consumption on which the power control has an effect, this gap represents $10 \%$ in the case of low capacity constraints. It increases and reaches around $35 \%$ of the average power consumption for a $5 \mathrm{Mbits} / \mathrm{s}$ capacity constraint. This accounts for $8 \%$ of the total base station's energy consumption. As a conclusion, the gap is not tremendous and the proposed policy has a good performance.

In order to better understand this gap, we display in Figures $5 \mathrm{~b}$ and $5 \mathrm{c}$ the average time during which a base station is active and the average transmit power when a base station serves users. In Figure 5b, we observe that the service time is nearly the same in the cases where the optimal and suboptimal policies are applied. On the contrary, Figure 5c shows that the RF transmit power is between 4 and 29 times higher where the sub-optimal solution is used.

\section{INTERFERENCE REDUCTION FOR PERFORMANCE IMPROVEMENT}

The results obtained in the previous section show that the performance of the proposed solution can be improved by mitigating the interference in the network. In [34], the authors present several solutions for reducing this interference. One of the limitations of some of them is that they require collaboration between the cells and consequently extra signaling. In this article, we prefer not to consider such collaborative solutions. That is why, we propose a simple, but efficient solution to enhance the power control policy introduced in the previous sections.

These results also suggest that a reduction of the RF transmit powers $\left(P_{T_{X}}\right)$ can efficiently reduce the network energy consumption. This can be done by reducing its maximum value, denoted $P_{\max }$. Indeed, reducing the maximum RF transmit power limits the value of the instantaneous transmit power and consequently the interference in the network.

In addition to improving the performance, the reduction of the maximum transmit power has the advantage of not requiring additional signaling. Indeed, we will see that the value of the maximum transmit power, for which the base station power consumption is minimum, depends little on the users' capacity constraints. More generally, this value depends little on the load of the base station. That is why, a good value for the maximum transmit power can be pre-configured and never changed.

We propose here to use the policy described in Section III with a reduced maximum transmit power where Cell 


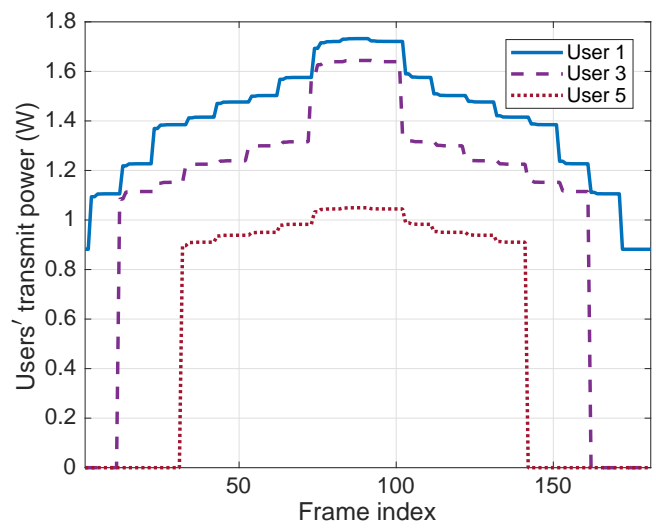

(a) Evolution of the instantaneous transmit power

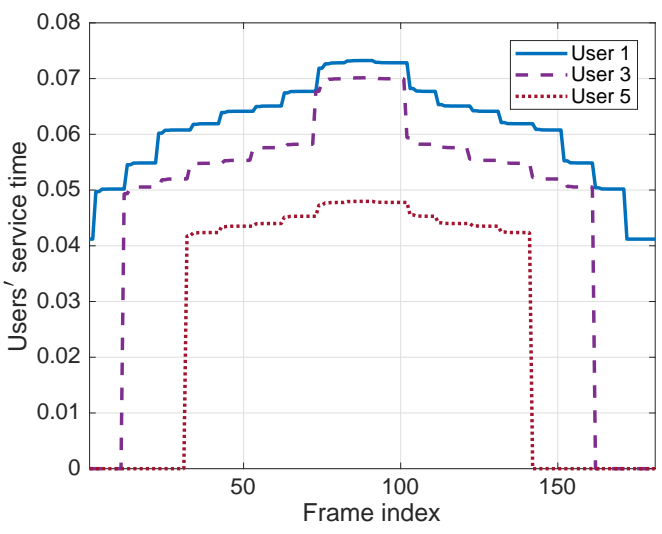

(b) Evolution of the users' proportion of time

Fig. 4. Evolution of the instantaneous transmit power of the base station and of the proportion of time during which each user is served when the users appear and disappear in the coverage area of the cells.

DTx is used. We denote $P_{\max }^{\mathrm{DTx}}$ the maximum base station transmit power with Cell DTx. Moreover, in order to avoid an increasing number of outages in the network, if the constraints of equation (8c) becomes binding, i.e., if the base station does not switch to sleep mode, the maximum transmit power is not reduced and is equal to $P_{\max }=6.3 \mathrm{~W}$.

In the following, we use approximations at low SINR and numerical simulations in order to show that the lower value of the maximum RF transmit power $P_{\max }^{\mathrm{DTx}}$ yields a performance closer to the optimal solution.

\section{A. Taylor Expansions at low SINR}

We derive, here, the optimal value of $P_{\max }^{\mathrm{DTx}}$ for low SINR values. According to Figure 3, in low SINR conditions, the users are served with the maximum transmit power $P_{\max }^{\mathrm{DTx}}$ during the minimum time $\mu_{n k}^{\min }$. Thereafter, we use the Taylor expansions $\log (1+x) \underset{x \approx 0}{=} x-\frac{x^{2}}{2}$ and $\frac{1}{1-x} \underset{x \approx 0}{=} 1+x$ to derive an approximation for $\mu_{n k}^{\min }$ :

$$
\begin{aligned}
\mu_{n k}^{\min }\left(\rho_{n k}^{M}\right) & =\frac{C_{k}}{B \log _{2}\left(1+\rho_{n k}^{M}\right)} \approx \frac{\ln (2) C_{k}}{B \rho_{n k}^{M}} \frac{1}{1-\frac{\rho_{n k}^{M}}{2}} \\
& \approx \frac{C_{k} \ln (2)}{B}\left[\frac{1}{\rho_{n k}^{M}}+\frac{1}{2}\right] .
\end{aligned}
$$

Moreover, as two base stations and a low SINR are considered here, if Cell DTx is used and the constraint of (8c) is not binding, the average base station's power consumption during a frame can be rewritten as:

$$
P_{\text {net }}=2 P_{s}+\left(P_{0}-P_{s}+m_{p} P_{\max }^{\mathrm{DTx}}\right)\left(\Sigma_{1}+\Sigma_{2}\right)
$$

where $\Sigma_{n}=\sum_{k \in S_{n}} \mu_{n k}$ for $n \in\{1 ; 2\}$. We approximate $\Sigma_{n}$ with equation (26) for $n \in\{1 ; 2\}$ and $m=1+(n \bmod 2)$ :

$$
\begin{aligned}
\Sigma_{n} & =\sum_{k \in S_{n}} \frac{C_{k}}{B \log _{2}\left(1+\frac{P_{\max }^{\mathrm{DTx}} G_{n k}}{N+G_{m k} P_{\max }^{\mathrm{DTx}} \Sigma_{m}}\right)} \\
& \approx\left[\frac{\gamma_{n}}{P_{\max }^{\mathrm{DTx}}}+\Sigma_{m} \delta_{n}+\epsilon_{n}\right],
\end{aligned}
$$

where $\gamma_{n}=\frac{N \ln (2)}{B} \sum_{k \in S_{n}} \frac{C_{k}}{G_{n k}}, \delta_{n}=\frac{\ln (2)}{B} \sum_{k \in S_{n}} \frac{G_{m k} C_{k}}{G_{n k}}$ and $\epsilon_{n}=\frac{\ln (2)}{2 B} \sum_{k \in S_{n}} C_{k}$. Replacing, $\Sigma_{m}$ by its expression, we derive:

$$
\Sigma_{n}=\frac{\frac{\gamma_{n}+\delta_{n} \gamma_{m}}{P_{\max }^{\operatorname{DT}}}+\epsilon_{n}+\epsilon_{m} \delta_{n}}{1-\delta_{n} \delta_{m}} .
$$

If we now insert equation (31) into equation (28), we finally derive the expression of the average base station's power consumption during a frame:

$$
\mathbb{E}\left[P_{\mathrm{net}}\right]=2 P_{s}+\left(P_{0}-P_{s}+m_{p} P_{\max }^{\mathrm{DTx}}\right)\left(\frac{1}{P_{\max }^{\mathrm{DTx}}} \mathbb{E}_{\gamma}+\mathbb{E}_{\epsilon}\right),
$$

where,

$$
\mathbb{E}_{\gamma}=\mathbb{E}\left[\frac{\gamma_{1}+\gamma_{2}+\delta_{1} \gamma_{2}+\delta_{2} \gamma_{1}}{1-\delta_{1} \delta_{2}}\right]
$$

and,

$$
\mathbb{E}_{\epsilon}=\mathbb{E}\left[\frac{\epsilon_{1}+\epsilon_{2}+\delta_{1} \epsilon_{2}+\delta_{2} \epsilon_{1}}{1-\delta_{1} \delta_{2}}\right] .
$$

Equation (32) is convex in $P_{\max }^{\mathrm{DTx}}$, we can consequently derive it to find the optimal value of $P_{\max }^{\mathrm{DTx}}$ for a low SINR value , which is denoted $P_{\max }^{\mathrm{DTx} *}$

$$
P_{\max }^{\mathrm{DTx} *}=\sqrt{\frac{\left(P_{0}-P_{s}\right) \mathbb{E}_{\gamma}}{m_{p} \mathbb{E}_{\epsilon}}} .
$$

The value $P_{\max }^{\mathrm{DTx} *}$ derived above can be evaluated using numerical simulations. In the network featuring two base stations and described in the previous sections, the value of $P_{\max }^{\mathrm{DTx} *}$ varies between 22.5 and $24 \mathrm{~mW}$, depending on the user's capacity constraint. This result, obtained at low SINR values, shows that the base station's power consumption is further reduced when $P_{\max }^{\mathrm{DTx}}$ is low.

\section{B. Simulation Results}

We now use numerical simulations in order to confirm our approximations for low SINR values. For that purpose, we consider the network composed of two base stations studied in the previous sections. As a first step, we display in Figure 6a the average energy consumption of the network for different values of $P_{\max }^{\mathrm{DTx}}$. This figure shows that reducing the maximum transmit power can greatly reduce the average base station's 


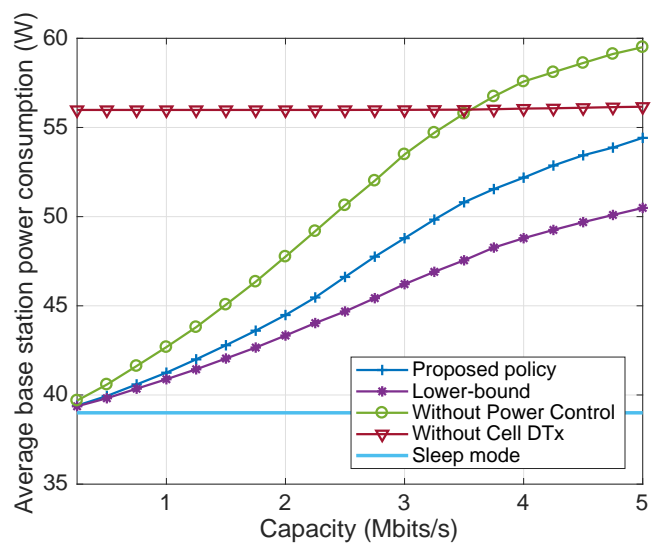

(a) Average base station power consumption

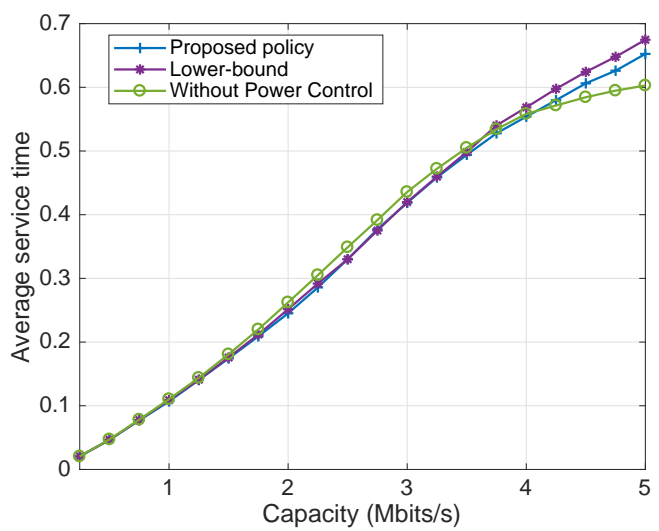

(b) Average time during which a base station is active

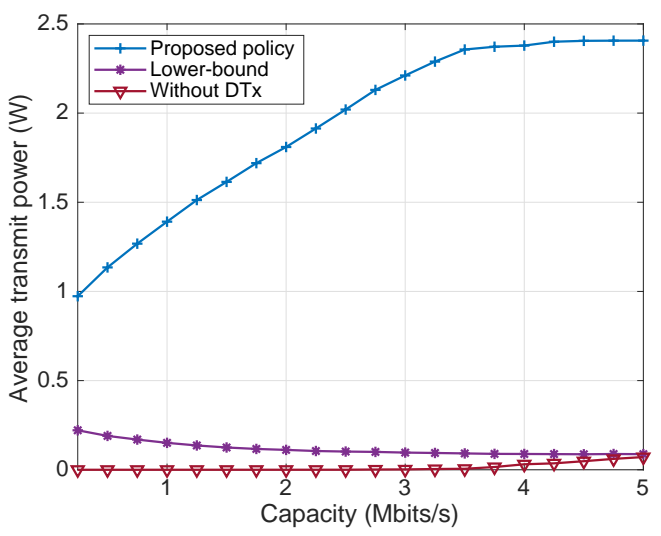

(c) Average transmit power

Fig. 5. Comparison of the average base station power consumption, service time and transmit power when the base station is active with four different policies. The proposed policy is compared with: a policy without power control, the optimal policy without Cell DTx and the lower-bound previously studied.

power consumption. Moreover, Figures $6 \mathrm{c}$ and $6 \mathrm{~b}$ show that reducing the maximum $\mathrm{RF}$ transmit power greatly reduces the average transmit power while increasing only slightly the total service time.

We now evaluate the value of $P_{\max }^{\mathrm{DTx}}$, for which the energy consumption is minimum. For this reason, we display in Figure 7 the evolution of the average base station's power consumption versus $P_{\max }^{\mathrm{DTx}}$, and this on a logarithmic scale for different values of the capacity constraint.

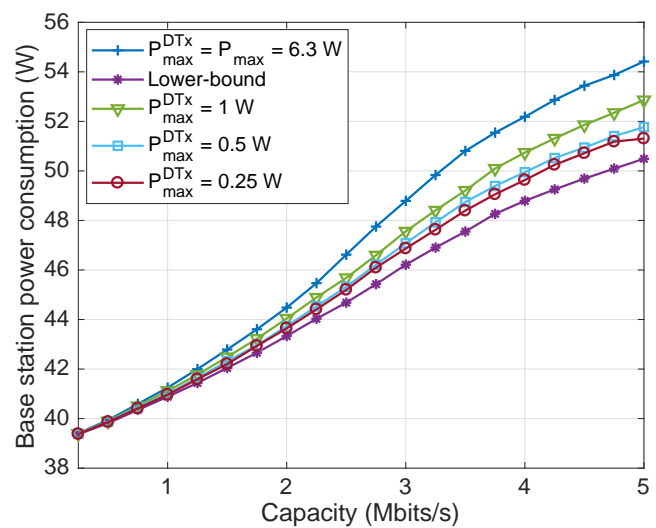

(a) Average base station power consumption

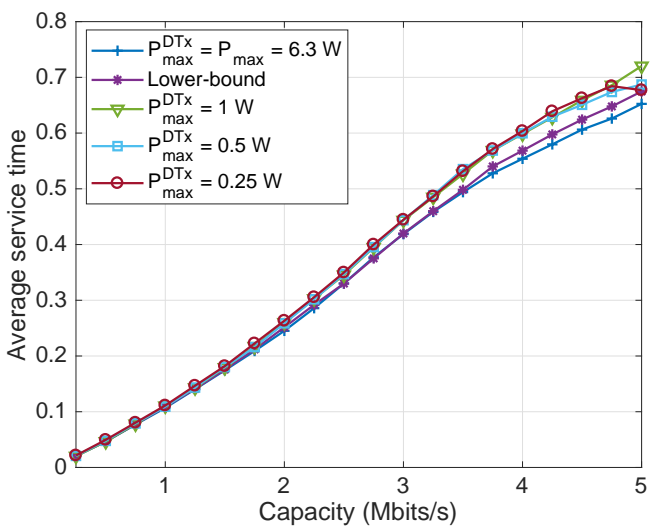

(b) Average service time

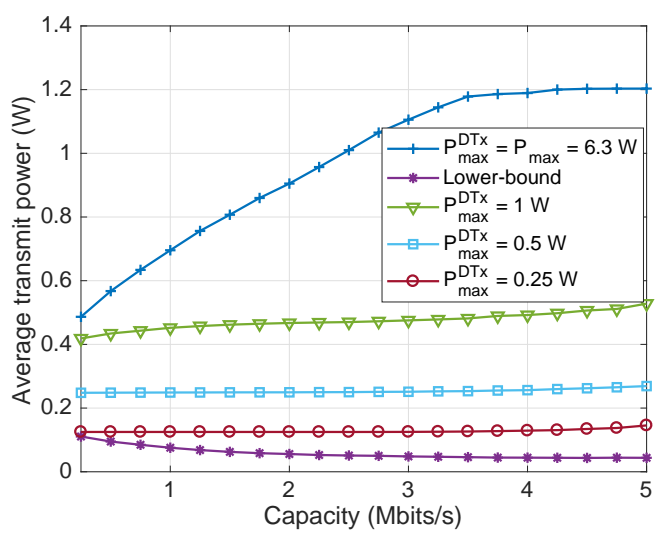

(c) Average transmit power

Fig. 6. Evolution of the average base station power consumption, service time and transmit power when the base station is active with a reduced maximum $\mathrm{RF}$ transmit power. We can see in this figure that reducing the maximum RF transmit power can reduce the average base station power consumption.

In these figures, the average base station's power consumption is minimized for about $P_{\max }^{\mathrm{DTx}}=150 \mathrm{~mW}$, for low capacity constraints. This optimal value decreases when the capacity constraint increases. As shown in Figure 7d, the optimal value of $P_{\max }^{\mathrm{DTx}}$ is equal to $50 \mathrm{~mW}$ for a capacity constraint equal to $5 \mathrm{Mbits} / \mathrm{s}$. It is worth noting that, for such a low value of the transmit power, and according to Figure 3, all users have to be served with the same transmit power equal to $P_{\max }^{\mathrm{DTx}}$.

As shown in Figure 7a, if the capacity constraint is low, the proposed policy is near optimal with any value of $P_{\max }^{\mathrm{DTx}}$. How- 


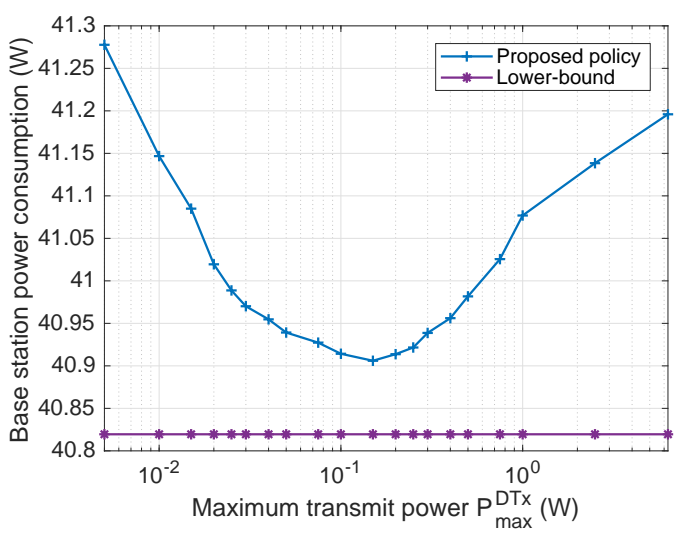

(a) $C=1 \mathrm{Mbits} / \mathrm{s}$

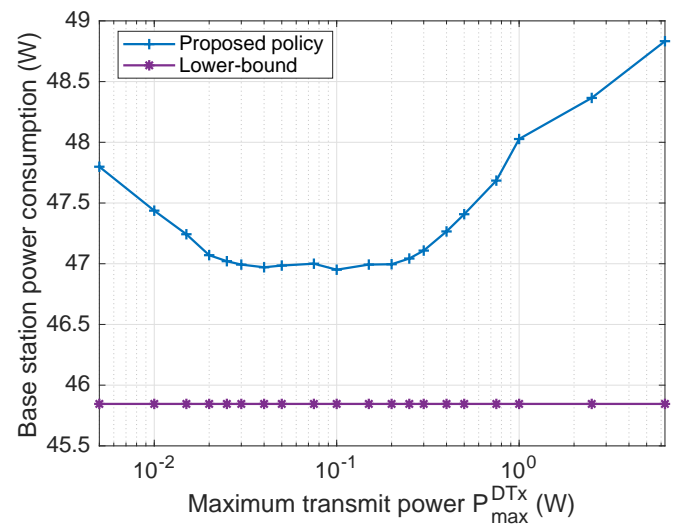

(c) $C=3 \mathrm{Mbits} / \mathrm{s}$

Fig. 7. Evolution of the average base station power consumption versus $P_{\max }^{\mathrm{DTX}}$

ever, given a capacity constraint of 5 Mbits/s, the reduction of the maximum transmit power reduces the gap between the optimal and sub-optimal strategies from $35 \%$ to $17.4 \%$, for the part of the power consumption on which power control has an effect. This reduction of the average power consumption represents $4 \%$ of the total base station's power consumption. Moreover, we can see that, for high values of the capacity constraint, i.e., when interference is high in the network, using a too low value of the maximum transmit power increases only slightly the average network's power consumption.

The purpose of computing an appropriate value for $P_{\max }^{\mathrm{DTx}}$ is to find the trade-off between the average static and dynamic network's power consumptions. When inter-cell interference is negligible, this trade-off can be computed thanks to the algorithm introduced in Section III. However, if interference is predominant, the dynamic power consumption has to be reduced in order to reduce interference. This result is in line with our low SINR approximation which shows that, for low SINR values, the average base station's power consumption is minimized for low values of $P_{\max }^{\mathrm{DTx}}$.

\section{EXTENSION TO DENSE NETWORKS}

In the previous sections, we conducted an analysis of the average base station's power consumption in a network comprising two base stations. In this section and the next one, we show that all our results, which were obtained with two base stations, remain valid in a dense network.

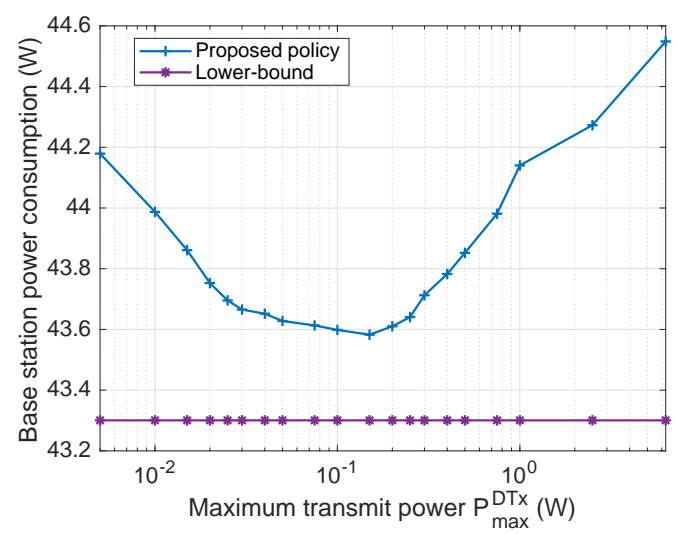

(b) $C=2 \mathrm{Mbits} / \mathrm{s}$

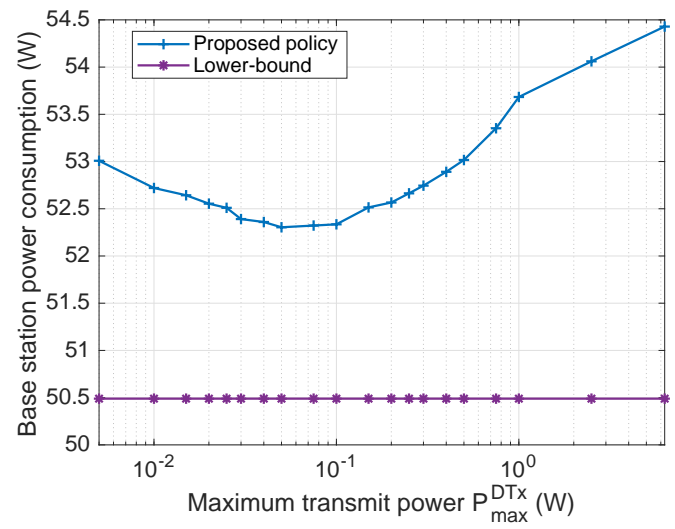

(d) $C=5 \mathrm{Mbits} / \mathrm{s}$

\section{A. Simulation Parameters}

In order to assess the performance of the proposed solution in a dense network, we consider the network featured in Figure 8. It is made of 17 hexagonal cells featuring a $500 \mathrm{~m}$ intersite distance. Mobile users are distributed following a Poisson Point Process and the user's density is equal to 20 users $/ \mathrm{km}^{2}$. Path-losses are computed thanks to the Winner II B1 channel model, similarly to the network's case studied in the previous sections. Each user is served by the base station that entails the lowest path-loss in a $10 \mathrm{MHz}$ band featuring a $2 \mathrm{GHz}$ central frequency. We suppose that all users have the same capacity constraint. Moreover, in order to avoid edge effects, we display only the average power consumption of the central base station.

\section{B. Behavior of the proposed policy in a dense network}

Before assessing the proposed solution in dense networks, its feasibility must be established. Indeed, when a new user connects to the network, the corresponding base station will increase its average transmit power and the time during which it is active. As a consequence, all the neighboring base stations will simultaneously increase their transmit power. Then, as explained in Section V-A, in the following frame, all the base stations will change their transmit power to cope with the additional interference and so on until the stabilization of both the transmit power and the users' service times. So, when a new user connects, there is a transition phase during 


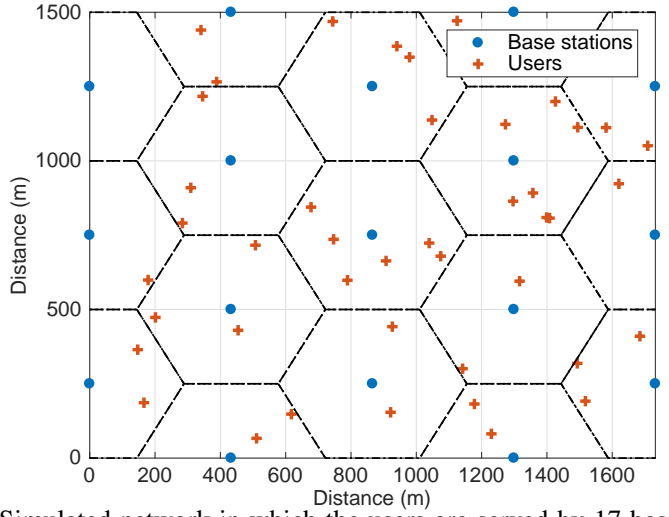

Fig. 8. Simulated network in which the users are served by 17 base stations The cells are hexagonal with a $500 \mathrm{~m}$ intersite distance.

which all the base stations adapt simultaneously their transmit power and service time. If this transition phase is too long, our solution cannot be applied in a dense network. The aim of this section is to assess the duration of this transition phase. To do so, we consider 30 users, who are indexed by their order of appearance, and we suppose that they connect to the network. A new user connects every ten frames and all the users have the same capacity constraint equal to $3 \mathrm{Mbits} / \mathrm{s}$. The results are displayed in Figures 9a and 9b.

We can observe in these figures that, when a new user connects to the network, the transition phase, during which the base stations update the transmit powers $\left(P_{T_{X}}\right)$ and service times $(\mu)$, last less than 5 frames. After this transition phase, the transmit power used for a user is constant. With such short transition phases, the proposed solution can be used in dense networks and the transition phases can be neglected in the evaluation of the base station's power consumption.

\section{Performance assessment}

Once the possibility to use the proposed solution in dense networks has been established, we can assess its performance. With more than two base stations, we cannot compare the performance of the proposed solution with the previously used lower-bound. In order to evaluate the proposed policy in a dense network, we compare the following policies:

- The proposed policy that features different values of $P_{\max }^{\mathrm{DTx}}$.

- The policy featuring no power control, in which all users are served during $\mu_{n k}^{\mathrm{min}}$, and where the transmit power is equal to $P_{\max }$.

- The optimal policy without Cell DTx.

The results are displayed in Figure 10a. This figure shows that the results obtained with two base stations remain valid when the number of base stations increases. In particular, the proposed power control policy provides better results than the policy without any power control and the policy without any Cell DTx. In the case of low capacity constraints, the proposed policy provides a $30 \%$ gain compared to the power control policy without Cell DTx. Moreover, without reduction of the maximum transmit power, this policy provides a gain of up to $6 \%$ compared to the policy featuring no power control. If we now analyze the gain provided by the reduction of the maximum transmit power $P_{\max }^{\mathrm{DTx}}$, we can see that, as in a network made of two cells, reducing $P_{\max }^{\mathrm{DTx}}$ thus reduces the base station's power consumption. Since the maximum transmit power is equal to $250 \mathrm{~mW}$, the gain in energy consumption represents up to $11 \%$ of the total base station's energy consumption, in comparison to the policy featuring no power control. Moreover, we can see in Figure 10c that, the average transmit power is greatly reduced when $P_{\max }^{\mathrm{DTx}}$ is reduced. Furthermore, Figure 10b shows that the average service time only slightly increases when the maximum transmit power is decreased.

\section{OPTIMAL MAXIMUM TRANSMIT POWER IN DENSE NETWORKS}

In the previous section, we have shown that, like in the two base stations' scenario, the performance of the proposed solution can be improved if the value of the maximum transmit power $P_{\max }^{\mathrm{DTx}}$ is reduced. In this section, we use the Taylor expansion at low SINR and numerical simulations, in order to analyze the value of $P_{\max }^{\mathrm{DTx}}$, which minimizes the energy consumption of the network.

\section{A. Taylor Expansions}

We extend here the derivation conducted in Section VI-A in a dense network. I.e., we use Taylor expansions, in order to derive the optimal value for $P_{\max }^{\mathrm{DTx}}$ for low SINR values. First of all, at low SINR values, and according to Figure 3, all users have to be served with the maximum transmit power $P_{\max }^{\mathrm{DTx}}$ during $\mu_{n k}^{\min }$. In such a case, the average network's power consumption can be expressed as:

$$
\mathbb{E}\left[P_{\mathrm{net}}\right]=N_{B S} P_{s}+\left(P_{0}-P_{s}+m_{p} P_{\max }^{\mathrm{DTx}}\right) \sum_{n=1}^{N_{B S}} \mathbb{E}\left[\Sigma_{n}\right],
$$

where $\Sigma_{n}=\sum_{k \in S_{n}} \mu_{n k}^{\min }$. Moreover, using the Taylor expansion of equation (26), we derive:

$$
\Sigma_{n}=\frac{\gamma_{n}}{P_{\max }^{\mathrm{DTx}}}+\sum_{m \neq n} \Sigma_{m} \delta_{n m}+\epsilon_{n}, \quad \forall n \in \llbracket 1 ; N_{B S} \rrbracket,
$$

where $\gamma_{n}=\frac{N \ln (2)}{B} \sum_{k \in S_{n}} \frac{C_{k}}{G_{n k}}, \delta_{n m}=\frac{\ln (2)}{B} \sum_{k \in S_{n}} \frac{C_{k} G_{m k}}{G_{n k}}$, and, $\epsilon_{n}=\frac{\ln (2)}{2 B} \sum_{k \in S_{n}} C_{k}$. Equation (37) provides a linear system with $N_{B S}$ equations in $\Sigma_{n}$. Its matrix equation can be written:

$$
\Delta \boldsymbol{\Sigma}=\frac{1}{P_{\max }^{\mathrm{DTx}}} \gamma+\boldsymbol{\epsilon}
$$

where, $\boldsymbol{\Sigma}=\left(\Sigma_{1}, \cdots, \Sigma_{N_{B S}}\right)^{T}, \gamma=\left(\gamma_{1}, \cdots, \gamma_{N_{B S}}\right)^{T}, \boldsymbol{\epsilon}=$ $\left(\epsilon_{1}, \cdots, \epsilon_{N_{B S}}\right)^{T}$ and, $\boldsymbol{\Delta}_{n m}=-\delta_{n m}$ if $m \neq n$ and $\boldsymbol{\Delta}_{n n}=1, \forall n \in$ $\llbracket 1 ; N_{B S} \rrbracket$. Once this linear system has been solved, we can rewrite the expression of the average base station's power consumption as follows:

$$
\begin{aligned}
\mathbb{E}\left[P_{\mathrm{net}}\right]= & N_{B S} P_{S}+\left(P_{0}-P_{S}+m_{p} P_{\max }^{\mathrm{DTx}}\right) \times \\
& \sum_{n=1}^{N_{B S}}\left(\frac{1}{P_{\max }^{\mathrm{DTx}}} \mathbb{E}\left[\left(\Delta^{-1} \gamma\right)_{n}\right]+\mathbb{E}\left[\left(\Delta^{-1} \boldsymbol{\epsilon}\right)_{n}\right]\right) .
\end{aligned}
$$




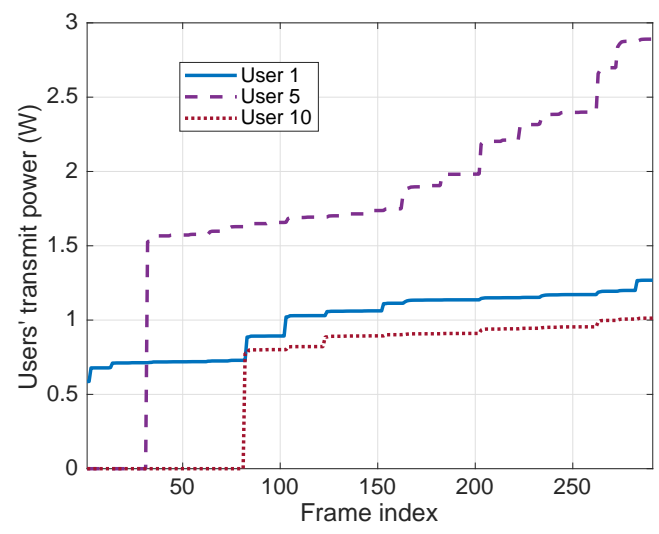

(a) Evolution of the instantaneous transmit power

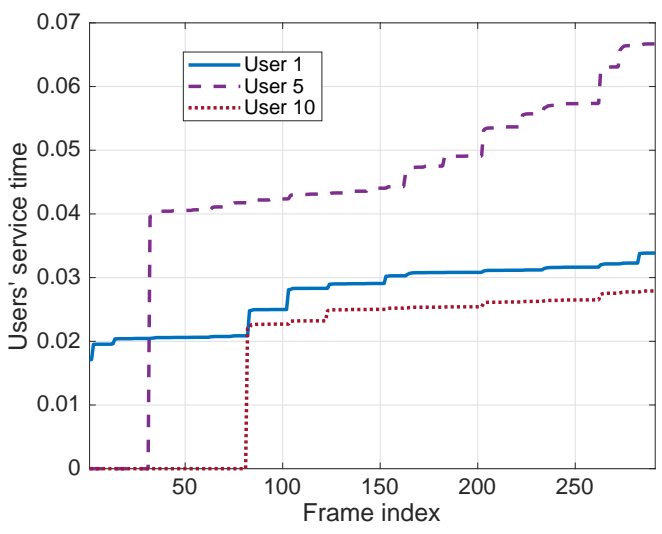

(b) Evolution of the users' proportion of time

Fig. 9. Evolution of the instantaneous transmit power and of the service time when users connects to the network.

We can finally derive the optimal value of $P_{\max }^{\mathrm{DTx}}$ thanks to a derivation of equation (39):

$$
P_{\max }^{\mathrm{DTx} *}=\sqrt{\frac{\left(P_{0}-P_{S}\right) \sum_{n=1}^{N_{B S}} \mathbb{E}\left[\left(\boldsymbol{\Delta}^{-1} \gamma\right)_{n}\right]}{m_{p} \sum_{n=1}^{N_{B S}} \mathbb{E}\left[\left(\boldsymbol{\Delta}^{-1} \boldsymbol{\epsilon}\right)_{n}\right]}}
$$

In the studied network, $P_{\max }^{\mathrm{DTx} *}$ is approximately equal to $11 \mathrm{~mW}$. As in the network with two base stations, for low SINR values, the base station's energy consumption is further reduced when the maximum transmit power $P_{\max }^{\mathrm{DTx}}$ is low.

\section{B. Simulation Results}

We now use numerical simulations in order to confirm that the base station's power consumption is optimal, where the maximum transmit power is low. We display in Figure 11 the evolution of the base station's power consumption versus $P_{\max }^{\mathrm{DTx}}$ on a logarithmic scale. We can see in this figure that, for low values of the capacity constraint, e.g., $1 \mathrm{Mbit} / \mathrm{s}$, reducing $P_{\max }^{\mathrm{DTx}}$ has little effect. On the contrary, with a higher capacity constraint, the base station's power consumption is high when $P_{\max }^{\mathrm{DTx}}=P_{\max }=6.3 \mathrm{~W}$. When $P_{\max }^{\mathrm{DTx}}$ decreases, the average power consumption sharply decreases until $P_{\max }^{\mathrm{DTx}}=400 \mathrm{~mW}$, then it continues to decrease and reaches a minimum value between $P_{\max }^{\mathrm{DTx}}=15 \mathrm{~mW}$ and $P_{\max }^{\mathrm{DTx}}=40 \mathrm{~mW}$. Finally, the average power consumption increases when $P_{\max }^{\mathrm{DTx}}$ becomes too low (below $10 \mathrm{~mW}$ ). Let us consider a $5 \mathrm{Mbits} / \mathrm{s}$ capacity constraint: in that case, reducing the maximum RF transmit power enables to save $33 \%$ of the power consumption, on which the power control has an effect. This represents $8 \%$ of the total average base station's power consumption.

This result shows that the base station's power consumption is minimized for low values of the maximum transmit power. For such low values of $P_{\max }^{\mathrm{DTx}}$, and within the framework of the proposed policy, all users have to be provided with the same transmit power that is equal to $P_{\max }^{\mathrm{DTx}}$.

In comparison with a network composed of two base stations, the number of neighboring users served by other base stations increases with the number of base stations. As a consequence, the interference is more significant. Consequently, the maximum transmit power has to be further reduced in order to mitigate the inter-cell interference.
Please, note that the optimal value of $P_{\max }^{\mathrm{DTx}}$ varies slightly with the users' capacity constraints. This shows that this value is only slightly dependent on the load of the base stations. As a consequence, the value of $P_{\max }^{\mathrm{DTx}}$ can be pre-configured and static. In other words, it does not have to be adapted to the network's load.

\section{CONCLUSION}

In this article, we study the problem of power control associated with Cell DTx in a multi-cell scenario. More precisely, we study the performance of a policy in which each base station optimizes its own energy consumption. For that purpose, we first consider a network composed of two base stations. In this network, we compute the minimum network's energy consumption and we use this minimum in order to assess the proposed policy. Our results show that the proposed policy has a good performance, which can be improved by reducing the maximum transmit power of the base station. Then we employ approximations at low SINR and numerical simulations in order to show that the best performance is obtained for low values of the transmit power. Finally, we use numerical simulations in a dense network in order to extend the analysis done in a two cells network. Our results show that the proposed policy reduces by up to $6 \%$ the energy consumption, in comparison with a policy without any power control. This gain can reach $11 \%$ when the base station's maximum transmit power is reduced. In our future work, we will investigate the problem of users' scheduling with Cell Dx in dense networks. We are also thinking of extending the results obtained in this article by considering either fading in the power control or multiple antennas both at the transmitter and the receiver.

\section{APPENDIX A}

\section{Optimal PROBLEM REFORMULATION}

In this appendix, we derive equation (22). In this proof, with no loss of generality, we suppose that $S_{1}=\llbracket 1 ; N_{1} \rrbracket$ and $S_{2}=\llbracket N_{1}+1 ; N_{u} \rrbracket$. First of all, the matrix equation of the linear system introduced by equation (21) is:

$$
\mathbf{M X}=\boldsymbol{\beta},
$$




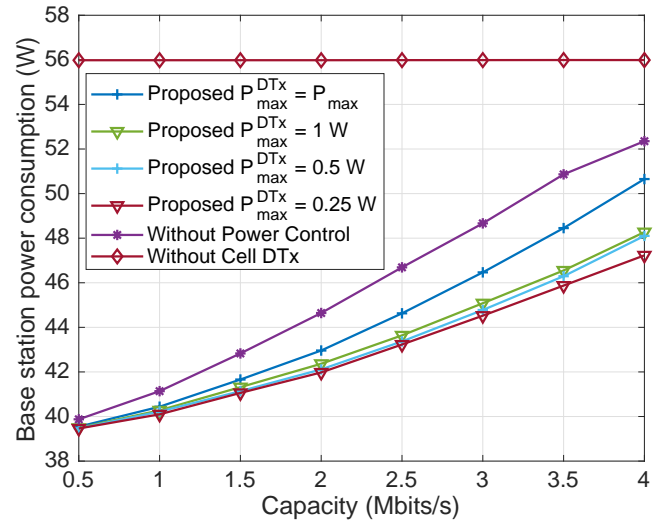

(a) Average base station power consumption

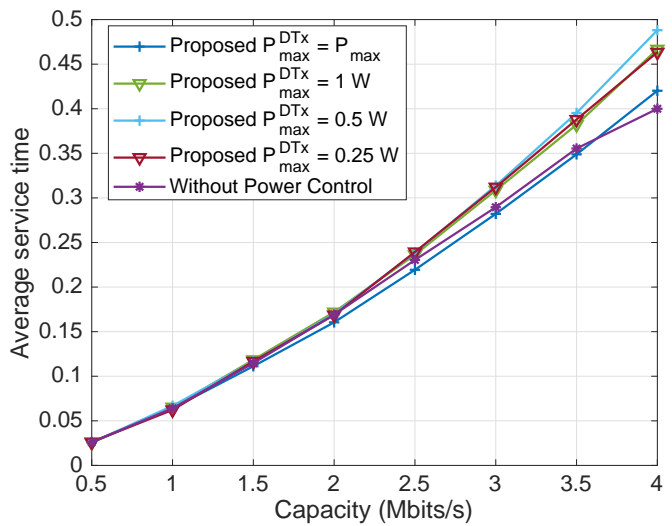

(b) Average service time

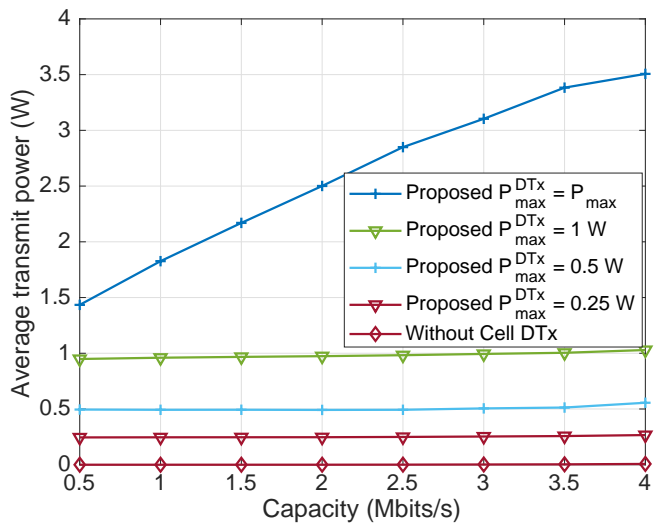

(c) Average transmit power

Fig. 10. Evolution of the average base station power consumption, time during which the base station is active and transmit power when the base station is active with a reduced maximum RF transmit power in a network composed of 17 base station.

where, $\boldsymbol{\beta}=\left(\beta_{1}, \cdots, \beta_{N_{u}}\right)^{T}, \boldsymbol{X}=\left(x_{1}, \cdots, x_{N_{u}}\right)^{T}$ and the matrix $\mathbf{M}$ can be written as the following block matrix:

$$
\mathbf{M}=\left(\begin{array}{c:c}
\mathbf{I}_{\mathbf{N}_{1}} & \boldsymbol{\Gamma}_{\mathbf{1}} \\
\hdashline \boldsymbol{\Gamma}_{\mathbf{2}} & \mathbf{I}_{\mathbf{N}_{2}}
\end{array}\right),
$$

where,

$$
\boldsymbol{\Gamma}_{\mathbf{1}}=\left(\begin{array}{ccc}
-\alpha_{1} & \cdots & -\alpha_{1} \\
\vdots & & \vdots \\
-\alpha_{N_{1}} & \cdots & -\alpha_{N_{1}}
\end{array}\right),
$$

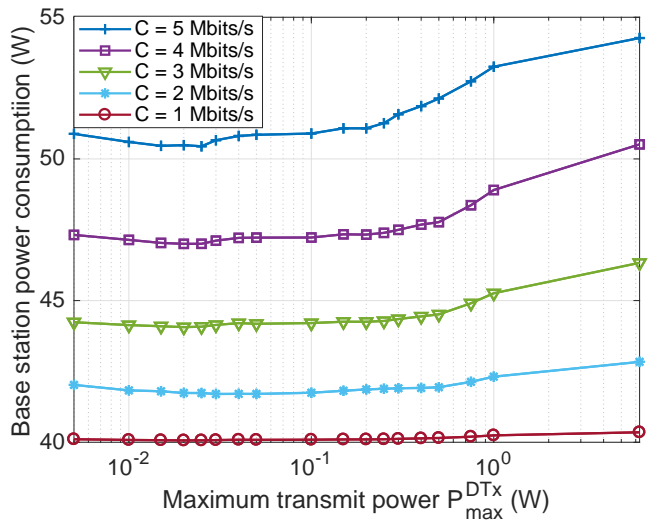

Fig. 11. Evolution of the average base station power consumption versus $P_{\max }^{\mathrm{DTT}}$ in the network composed of 17 base stations displayed in Figure 8.

and,

$$
\boldsymbol{\Gamma}_{2}=\left(\begin{array}{ccc}
-\alpha_{N_{1}+1} & \cdots & -\alpha_{N_{1}+1} \\
\vdots & & \vdots \\
-\alpha_{N_{u}} & \cdots & -\alpha_{N_{u}}
\end{array}\right) .
$$

Moreover, $\mathbf{I}_{\mathbf{N}_{1}}$ and $\mathbf{I}_{\mathbf{N}_{\mathbf{2}}}$ denote respectively the identity matrix of size $N_{1}$ and $N_{2}$.

Furthermore, the matrices $\boldsymbol{\Gamma}_{\mathbf{1}} \in M_{N_{1} \times N_{2}}(\mathbb{R})$ and $\boldsymbol{\Gamma}_{\mathbf{2}} \in$ $M_{N_{2} \times N_{1}}(\mathbb{R})$ are not square matrices. In the following, we also use the matrices $\Gamma_{1}^{\prime} \in M_{N_{1}}(\mathbb{R})$ and $\Gamma_{2}^{\prime} \in M_{N_{2}}(\mathbb{R})$ whose columns are respectively equal to the columns of $\boldsymbol{\Gamma}_{\mathbf{1}}$ and $\boldsymbol{\Gamma}_{\mathbf{2}}$. The matrix of the system is a block matrix whose inverse can be computed with the following formula [35]:

$$
\left(\begin{array}{ll}
\mathbf{A} & \mathbf{B} \\
\mathbf{C} & \mathbf{D}
\end{array}\right)^{-1}=\left(\begin{array}{cc}
\mathbf{A}^{-1}+\mathbf{A}^{-1} \mathbf{B E C A}^{-1} & -\mathbf{A}^{-1} \mathbf{B E} \\
-\mathbf{E} \mathbf{C} \mathbf{A}^{-1} & \mathbf{E}
\end{array}\right),
$$

where,

$$
\mathbf{E}=\left(\mathbf{D}-\mathbf{C A}^{-1} \mathbf{B}\right)^{-1} .
$$

From equation (45), we derive:

$\mathbf{M}^{-1}=\left(\begin{array}{cc}\mathbf{I}_{\mathbf{N}_{1}}+\boldsymbol{\Gamma}_{\mathbf{1}}\left(\mathbf{I}_{\mathbf{N}_{2}}-\boldsymbol{\Gamma}_{2} \boldsymbol{\Gamma}_{1}\right)^{-1} \boldsymbol{\Gamma}_{2} & -\boldsymbol{\Gamma}_{\mathbf{1}}\left(\mathbf{I}_{\mathbf{N}_{2}}-\boldsymbol{\Gamma}_{2} \boldsymbol{\Gamma}_{1}\right)^{-1} \\ -\left(\mathbf{I}_{\mathbf{N}_{2}}-\boldsymbol{\Gamma}_{\mathbf{2}} \boldsymbol{\Gamma}_{\mathbf{1}}\right)^{-1} \boldsymbol{\Gamma}_{\mathbf{2}} & \left(\mathbf{I}_{\mathbf{N}_{2}}-\boldsymbol{\Gamma}_{\mathbf{2}} \boldsymbol{\Gamma}_{\mathbf{1}}\right)^{-1}\end{array}\right)$.

Furthermore, with the expressions of $\boldsymbol{\Gamma}_{\mathbf{1}}$ and $\boldsymbol{\Gamma}_{\mathbf{2}}$, we derive:

$$
\boldsymbol{\Gamma}_{\mathbf{2}} \boldsymbol{\Gamma}_{\mathbf{1}}=\operatorname{Tr}\left(\boldsymbol{\Gamma}_{1}^{\prime}\right) \boldsymbol{\Gamma}_{2}^{\prime}
$$

where $\operatorname{Tr}($.$) denotes the trace of the matrix. Consequently,$

$$
\left(\boldsymbol{\Gamma}_{\mathbf{2}} \boldsymbol{\Gamma}_{\mathbf{1}}\right)^{2}=\operatorname{Tr}\left(\boldsymbol{\Gamma}_{\mathbf{2}} \boldsymbol{\Gamma}_{\mathbf{1}}\right) \boldsymbol{\Gamma}_{\mathbf{2}} \boldsymbol{\Gamma}_{\mathbf{1}}
$$

This proves that:

$$
\begin{array}{r}
\left(\mathbf{I}_{\mathbf{N}_{2}}-\boldsymbol{\Gamma}_{2} \boldsymbol{\Gamma}_{1}\right)\left(\mathbf{I}_{\mathbf{N}_{2}}+\frac{1}{1-\operatorname{Tr}\left(\boldsymbol{\Gamma}_{2} \boldsymbol{\Gamma}_{\mathbf{1}}\right)} \boldsymbol{\Gamma}_{\mathbf{2}} \boldsymbol{\Gamma}_{\mathbf{1}}\right) \\
=\left(\mathbf{I}_{\mathbf{N}_{2}}+\frac{1}{1-\operatorname{Tr}\left(\boldsymbol{\Gamma}_{2} \boldsymbol{\Gamma}_{1}\right)} \boldsymbol{\Gamma}_{2} \boldsymbol{\Gamma}_{\mathbf{1}}\right)\left(\mathbf{I}_{\mathbf{N}_{2}}-\boldsymbol{\Gamma}_{2} \boldsymbol{\Gamma}_{\mathbf{1}}\right)=\mathbf{I}_{\mathbf{N}_{2}} .
\end{array}
$$

Consequently,

$$
\left(\mathbf{I}_{\mathbf{N}_{2}}-\boldsymbol{\Gamma}_{\mathbf{2}} \boldsymbol{\Gamma}_{\mathbf{1}}\right)^{-1}=\mathbf{I}_{\mathbf{N}_{\mathbf{2}}}+\frac{\operatorname{Tr}\left(\boldsymbol{\Gamma}_{\mathbf{1}}^{\prime}\right)}{1-\operatorname{Tr}\left(\boldsymbol{\Gamma}_{\mathbf{2}}^{\prime}\right) \operatorname{Tr}\left(\boldsymbol{\Gamma}_{\mathbf{1}}^{\prime}\right)} \boldsymbol{\Gamma} \mathbf{2}^{\prime} .
$$

We can use this expression to derive the expression of $M^{-1}$ :

$\mathbf{M}^{-1}=\left(\begin{array}{cc}\mathbf{I}_{\mathbf{N}_{\mathbf{1}}}+\frac{\operatorname{Tr}\left(\boldsymbol{\Gamma}_{\mathbf{2}}^{\prime}\right)}{1-\operatorname{Tr}\left(\boldsymbol{\Gamma}_{\mathbf{2}}^{\prime}\right) \operatorname{Tr}\left(\boldsymbol{\Gamma}_{\mathbf{1}}^{\prime}\right)} \boldsymbol{\Gamma}_{\mathbf{1}}^{\prime} & \frac{-1}{1-\operatorname{Tr}\left(\boldsymbol{\Gamma}_{\mathbf{2}}^{\prime}\right) \operatorname{Tr}\left(\boldsymbol{\Gamma}_{\mathbf{1}}^{\prime}\right)} \boldsymbol{\Gamma}_{\mathbf{1}} \\ \frac{-1}{1-\operatorname{Tr}\left(\boldsymbol{\Gamma}_{\mathbf{2}}^{\prime}\right) \operatorname{Tr}\left(\boldsymbol{\Gamma}_{\mathbf{1}}^{\prime}\right)} \boldsymbol{\Gamma}_{\mathbf{2}} & \mathbf{I}_{\mathbf{N}_{\mathbf{2}}}+\frac{\operatorname{Tr}\left(\boldsymbol{\Gamma}_{\mathbf{1}}^{\prime}\right)}{1-\operatorname{Tr}\left(\boldsymbol{\Gamma}_{\mathbf{2}}^{\prime}\right) \operatorname{Tr}\left(\boldsymbol{\Gamma}_{\mathbf{1}}^{\prime}\right)} \boldsymbol{\Gamma}_{\mathbf{2}}^{\prime}\end{array}\right)$ 

(21):

Finally, we solve the linear system introduced in equation

$$
\mathbf{X}=\mathbf{M}^{-1} \boldsymbol{\beta} \text {. }
$$

For all $k \in \llbracket 1 ; N_{u} \rrbracket$, denoting $m=1+(n \bmod 2)$, we derive:

$$
x_{k}=\beta_{k}+\alpha_{k}\left[\frac{\sum_{i \in S_{n}} \sum_{j \in S_{m}} \alpha_{j} \beta_{i}+\sum_{j \in S_{m}} \beta_{j}}{1-\sum_{i \in S_{n}} \sum_{j \in S_{m}} \alpha_{i} \alpha_{j}}\right] .
$$

\section{APPENDIX B}

\section{CONVEXity OF The Optimal STRATEGY}

In this appendix, we prove Proposition IV.1. As a first remark, in the problem of (25), inequalities of equations (25c) and (25d) are strict inequalities. According to [32], a convex problem should have only large inequalities. With these two strict inequalities, the problem cannot be convex. However, these strict inequalities can easily be replaced by large inequalities without losing the optimality by introducing $\epsilon \ll 1$. These constraints can be written:

$$
\begin{gathered}
\sum_{i \in S_{1}} \sum_{j \in S_{2}} \alpha_{i} \alpha_{j} \leq 1-\epsilon, \\
\mu_{n k} \geq \epsilon, \quad \forall k \in \llbracket 1 ; N_{u} \rrbracket .
\end{gathered}
$$

By choosing a sufficiently small $\epsilon$, the transformation of strict inequalities in large inequalities does not change the solution provided by the resolution of the problem.

We can now prove the convexity of the problem of equation (25). In this proof, with no loss of generality, we suppose that $S_{1}=\llbracket 1 ; N_{1} \rrbracket$ and $S_{2}=\llbracket N_{1}+1 ; N_{u} \rrbracket$. Moreover, in order to prove the convexity of the problem, we shall prove the convexity of the set defined by the constraints and that of the objective function. This can be done proving that $x_{k}$ are convex functions of $\mu_{n k}$ for $\mu_{n k} \in(0 ; 1], \forall k \in \llbracket 1 ; N_{u} \rrbracket$. Our proof relies on the following lemma:

Lemma B.1. The function $f(x)=\ln \left(x\left(e^{1 / x}-1\right)\right)$ is convex for $x \in \mathbb{R}_{+}^{*}$, the set of strictly positive real numbers.

Proof. The derivative of the function $f$ for $x>0$ is:

$$
f^{\prime}(x)=-\frac{1}{x}\left(\frac{1}{x\left(1-e^{\frac{-1}{x}}\right)}-1\right)=-\frac{1}{x} g(x) .
$$

In order to prove that $f$ is convex, we prove that its derivative is increasing. For that purpose, we first study the function $g$. First of all, the inequality:

$$
e^{x}>1+x, \quad \forall x \in \mathbb{R}^{*},
$$

proves that $g(x)>0, \forall x>0$. In equation (59), $\mathbb{R}^{*}$ denotes the set of non-zero real numbers.

Then, the derivative of $x\left(1-e^{\frac{-1}{x}}\right)$ is:

$$
\left(x\left(1-e^{\frac{-1}{x}}\right)\right)^{\prime}=1-e^{\frac{-1}{x}}-\frac{e^{\frac{-1}{x}}}{x} .
$$

Furthermore, inequality (59) shows that this derivative is strictly positive. Thus $g$ is a decreasing function as the inverse of an increasing function. Then $\frac{g(x)}{x}$ is decreasing over $\mathbb{R}_{+}^{*}$ as the product of two positive and decreasing functions. Finally, $f^{\prime}$ is increasing as the opposite of a decreasing function. Thus $f$ is convex.
Lemma B.1 proves that all the functions $\alpha_{k}, \beta_{k}$ are log-convex for all $k$. As a consequence, every product of these functions is convex [32]. Furthermore, since $\sum_{i=1}^{N_{1}} \sum_{j=N_{1}+1}^{N_{u}} \alpha_{i} \alpha_{j} \in(0 ; 1)$, we use the following Taylor series:

$$
\frac{1}{1-x}=\sum_{n=0}^{+\infty} x^{n}, \quad \forall x \in[0 ; 1) .
$$

In the rest of this article, in order to improve the readability, we denote:

$$
\sum_{l=1}^{N_{1} N_{2}} \gamma_{l}=\sum_{i=1}^{N_{1}} \sum_{j=N_{1}+1}^{N_{u}} \alpha_{i} \alpha_{j}
$$

where, $\gamma_{l}=\alpha_{(l-1)\left(\bmod N_{1}\right)+1} \times \alpha_{N_{1}+\left\lfloor\frac{l-1}{N_{1}}\right\rfloor+1}$, and $\lfloor$.$\rfloor denotes$ the floor function. Each function $\gamma_{l}$ is log-convex as the product of log-convex functions. With the Tayor series of equation (61), the expression of $x_{k}$ can be rewritten:

$$
x_{k}=\beta_{k}+\alpha_{k}\left[\sum_{i=1}^{N_{1}} \sum_{j=N_{1}+1}^{N_{u}} \alpha_{j} \beta_{i}+\sum_{j=N_{1}+1}^{N_{u}} \beta_{j}\right]\left[\sum_{n=0}^{+\infty}\left(\sum_{l=1}^{N_{1} N_{2}} \gamma_{l}\right)^{n}\right] \text {. }
$$

Then, using the multinomial coefficients [36], we derive:

$$
\begin{aligned}
x_{k}=\beta_{k}+\sum_{n=0}^{+\infty}[ & \left(\sum_{i=1}^{N_{1}} \sum_{j=N_{1}+1}^{N_{u}} \alpha_{j} \beta_{i} \alpha_{k}+\sum_{j=N_{1}+1}^{N_{u}} \alpha_{k} \beta_{j}\right) \times \\
& \left.\left(\sum_{\sum n_{l}=n}\left(\begin{array}{c}
n \\
n_{1}, \ldots, n_{N_{1}} N_{2}
\end{array}\right) \prod_{l=1}^{N_{1} N_{2}} \gamma_{l}^{n_{l}}\right)\right] .
\end{aligned}
$$

Each product $\prod_{l=1}^{N_{1} N_{2}} \gamma_{l}^{n_{l}}$ is log-convex as the product of long-convex functions. Consequently, expanding the product in the infinite sum, we express the terms as sums of product of log-convex functions. These products are consequently logconvex functions. Finally, an infinite sum of convex function is convex [32]. Which proves that $x_{k}$ is convex for all $k$. As a consequence, the studied problem is convex.

\section{REFERENCES}

[1] A. Fehske, G. Fettweis, J. Malmodin, and G. Biczok, "The global footprint of mobile communications: The ecological and economic perspective," IEEE Communications Magazine, vol. 49, pp. 55-62, August 2011

[2] I. Ashraf, F. Boccardi, and L. Ho, "SLEEP mode techniques for small cell deployments," IEEE Communications Magazine, vol. 49, pp. 72-79, August 2011.

[3] J. Wu, Y. Zhang, M. Zukerman, and E. K. N. Yung, "Energy-Efficient Base-Stations Sleep-Mode Techniques in Green Cellular Networks: A Survey," IEEE Communications Surveys Tutorials, vol. 17, pp. 803-826, Secondquarter 2015.

[4] M. Peng, Y. Li, J. Jiang, J. Li, and C. Wang, "Heterogeneous cloud radio access networks: a new perspective for enhancing spectral and energy efficiencies," IEEE Wireless Communications, vol. 21, pp. 126135, December 2014

[5] S. Samarakoon, M. Bennis, W. Saad, and M. Latva-aho, "Opportunistic sleep mode strategies in wireless small cell networks," in 2014 IEEE International Conference on Communications (ICC), pp. 2707-2712, June 2014.

[6] L. Suarez and L. Nuaymi, "Multi-size cell expansion for energy-efficient cell breathing in green wireless networks," in 2015 IEEE 82nd Vehicular Technology Conference (VTC2015-Fall), pp. 1-5, Sept 2015.

[7] T. Chen, H. Zhang, Z. Zhao, and X. Chen, "Towards green wireless access networks," in 2010 5th International ICST Conference on Communications and Networking in China, pp. 1-6, Aug 2010. 
[8] P. Frenger, P. Moberg, J. Malmodin, Y. Jading, and I. Godor, "Reducing Energy Consumption in LTE with Cell DTX," in 2011 IEEE 73rd Vehicular Technology Conference (VTC Spring), pp. 1-5, May 2011.

[9] A. Chatzipapas, S. Alouf, and V. Mancuso, "On the minimization of power consumption in base stations using on/off power amplifiers," in 2011 IEEE Online Conference on Green Communications, pp. 18-23, Sept 2011

[10] T. Chen, Y. Yang, H. Zhang, H. Kim, and K. Horneman, "Network energy saving technologies for green wireless access networks," IEEE Wireless Communications, vol. 18, pp. 30-38, October 2011.

[11] D. Kivanc, G. Li, and H. Liu, "Computationally efficient bandwidth allocation and power control for OFDMA," IEEE Transactions on Wireless Communications, vol. 2, pp. 1150-1158, Nov 2003.

[12] D. Lopez-Perez and al., "Power Minimization Based Resource Allocation for Interference Mitigation in OFDMA Femtocell Networks," IEEE Journal on Selected Areas in Communications, vol. 32, pp. 333-344, February 2014.

[13] H. Holtkamp, G. Auer, and H. Haas, "On Minimizing Base Station Power Consumption," in 2011 IEEE Vehicular Technology Conference (VTC Fall), pp. 1-5, Sept 2011.

[14] R. Wang and al., "Sleep mode design for green base stations," IET Communications, vol. 5, pp. 2606-2616, Dec 2011.

[15] R. M. Corless, G. H. Gonnet, D. E. G. Hare, D. J. Jeffrey, and D. E. Knuth, "On the Lambert W Function," in Advances in computational mathematics, pp. 329-359, 1996.

[16] R. Bonnefoi, C. Moy, and J. Palicot, Dynamic Sleep Mode for Minimizing a Femtocell Power Consumption, pp. 618-629. Cham: Springer International Publishing, 2016.

[17] R. Bonnefoi, C. Moy, and J. Palicot, "New Macrocell Downlink Energy Consumption Minimization with Cell DTx and Power Control," in IEEE International Conference on Communications, pp. 1-7, May 2017.

[18] H. Holtkamp, G. Auer, S. Bazzi, and H. Haas, "Minimizing Base Station Power Consumption," IEEE Journal on Selected Areas in Communications, vol. 32, pp. 297-306, February 2014.

[19] H. B. Ren, M. Zhao, J. K. Zhu, and W. Y. Zhou, "Energy-efficient resource allocation for OFDMA networks with sleep mode," Electronics Letters, vol. 49, pp. 111-113, January 2013.

[20] R.Bonnefoi, C. Moy, and J. Palicot, "Power Allocation for Minimizing Energy Consumption of OFDMA Downlink with Cell DTx," in 2017 24th International Conference on Telecommunications (ICT), pp. 1-7, May 2017.

[21] P. Chang and G. Miao, "Joint Optimization of Base Station DeepSleep and DTX Micro-Sleep," in 2016 IEEE Globecom Workshops (GC Wkshps), pp. 1-6, Dec 2016.

[22] P. Chang and G. Miao, "Energy and Spectral Efficiency of Cellular Networks with Discontinuous Transmission," IEEE Transactions on Wireless Communications, vol. PP, no. 99, pp. 1-1, 2017.

[23] W. Nie, Y. Zhong, F. C. Zheng, W. Zhang, and T. O'Farrell, "HetNets With Random DTX Scheme: Local Delay and Energy Efficiency," IEEE Transactions on Vehicular Technology, vol. 65, pp. 6601-6613, Aug 2016.

[24] A. D. Domenico, R. Gupta, and E. C. Strinati, "Dynamic Traffic Management for Green Open Access Femtocell Networks," in 2012 IEEE 75th Vehicular Technology Conference (VTC Spring), pp. 1-6, May 2012.

[25] R. Gupta, E. C. Strinati, and D. Ktenas, "Energy efficient joint DTX and MIMO in cloud Radio Access Networks," in 2012 IEEE 1st International Conference on Cloud Networking (CLOUDNET), pp. 191196, Nov 2012.

[26] S. Tombaz, S. w. Han, K. W. Sung, and J. Zander, "Energy Efficient Network Deployment With Cell DTX," IEEE Communications Letters, vol. 18, pp. 977-980, June 2014

[27] G. Andersson, A. Vastberg, A. Devlic, and C. Cavdar, "Energy efficient heterogeneous network deployment with cell DTX," in 2016 IEEE International Conference on Communications (ICC), pp. 1-6, May 2016.

[28] A. Cheng, H. Jin, J. Li, Y. Yu, and M. Peng, "Joint discontinuous transmission and power control for high energy efficiency in heterogeneous small cell networks," in 2014 IEEE 25th Annual International Symposium on Personal, Indoor, and Mobile Radio Communication (PIMRC), pp. 970-975, Sept 2014.

[29] S. Wu, F. Liu, Z. Zeng, and H. Xia, "Cooperative Sleep and Power Allocation for Energy Saving in Dense Small Cell Networks," IEEE Access, vol. 4, pp. 6993-7004, 2016.

[30] G. Auer and al., "How much energy is needed to run a wireless network?," IEEE Wireless Communications, vol. 18, pp. 40-49, October 2011.
[31] J. D. Hoffman, Numerical methods for engineers and scientists. New York, NY: McGraw-Hill, 1992.

[32] S. Boyd and L. Vandenberghe, Convex Optimization. New York, NY, USA: Cambridge University Press, 2004.

[33] P. Kyosti and al., "Winner II Deliverable 1.1.2.: Winner II Channel Models," tech. rep., September 2007.

[34] N. Saquib, E. Hossain, L. B. Le, and D. I. Kim, "Interference management in ofdma femtocell networks: issues and approaches," IEEE Wireless Communications, vol. 19, pp. 86-95, June 2012.

[35] D. S. Bernstein, Matrix mathematics : theory, facts, and formulas with application to linear systems theory. Princeton University Press.

[36] “NIST Digital Library of Mathematical Functions." http://dlmf.nist.gov/, Release 1.0.18 of 2018-03-27. F. W. J. Olver, A. B. Olde Daalhuis, D. W. Lozier, B. I. Schneider, R. F. Boisvert, C. W. Clark, B. R. Miller and B. V. Saunders, eds.

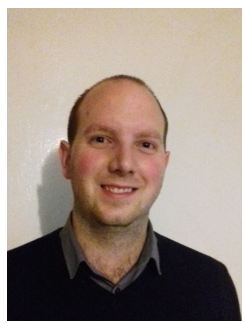

Rémi Bonnefoi received an engineering degree in telecommunication and electrical engineering from Supélec and a M.Sc. in electrical engineering from Université de Rennes 1 in 2015 . He is currently a third year PhD student in the SCEE reseach team at CentraleSupélec/IETR. His research interests are IoT networks, MIMO systems, green communications, cognitive radio and the aim of is $\mathrm{PhD}$ is to reduce the carbon footprint of mobile networks.

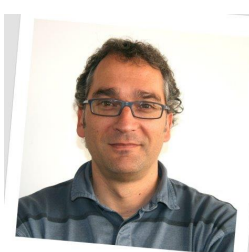

Christophe Moy is a Professor with University of Rennes 1 where he has been teaching in ISTIC syllabus since 2017. His research has been done since 2005 in IETR entity of CNRS (UMR 6164) where he focuses on Software Radio, Cognitive Radio (learning for dynamic spectrum access - DSA) and Green Radio. He was involved in many European and French collaborative projects: NoE NEWCOM and NEWCOM++, E\&sup2;R, EULER, E\&sup2;Rphase2, A3S, Mopcom, Idromel, WiNoCoD, "pole I\&R" SoftRF, CominLabs TEPN. He is a member of IRT B-COM. He is the head of the Signal and Communication Department of IETR. Christophe MOY received his Eng., M.Sc. and Ph.D. degrees in Electronics in 1995 and 1999 from the INSA of Rennes, France. He then worked 6 years at Mitsubishi Electric ITE-TCL research lab where he inititated studies on Software Radio systems and concepts. Then he was a Professor with Supelec between 2005 and 2014, and with CentraleSupelec from 2015 to 2017 . 


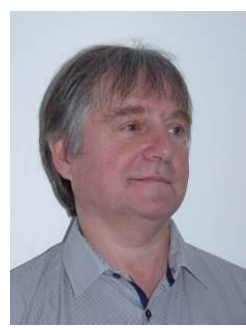

Jacques Palicot received, in 1983, his $\mathrm{PhD}$ degree in Signal Processing from the University of Rennes. Since 1988, he has been involved in studies about equalization techniques applied to digital transmissions and analog TV systems. Since 1991 he has been involved mainly in studies concerning the digital communications area and automatic measurements techniques. He has taken an active part in various international bodies EBU, CCIR, URSI, and within RACE, ACTS and IST European projects. He has published various scientific articles notably on equalization techniques, echo cancellation, hierarchical modulations and Software Radio techniques. He is author or co-author of more than 300 publications with more than 50 in journals, two books and 22 patents. $\mathrm{He}$ is currently involved in adaptive Signal Processing, digital communications,Software Radio, Cognitive radio and Green Radio. From November 2001 to September 2003 he had a temporary position with INRIA/IRISA in Rennes. He serves as Associate Editor for EURASIP JASP since 2008. He also served as lead guest editor for several Special Issues on Software Radio, Cognitive Radio and Green Radio. He was Co General Chairman of ISCIT 2011, Co General Chairman of Next-GWiN 2014, Technical Program Chairman of CROWNCOM 2009, Technical Program Chairman of GREENCOM 2013 and Technical Program Chairman of CRN Symposium of ICC 2014. Since October 2003 he is with CentraleSupélec in Rennes where he leads the Signal Communications and Embedded Electronics (SCEE) research team. 